\title{
First Infilling of the Venda Nova II Unlined High-Pressure Tunnel: Observed Behaviour and Numerical Modelling
}

\author{
Luís N. Lamas · Noemí S. Leitão · Carlos Esteves • \\ Nadir Plasencia
}

Received: 26 July 2012/Accepted: 12 March 2013/Published online: 11 April 2013

(c) Springer-Verlag Wien 2013

\begin{abstract}
The underground structures of the Venda Nova II reversible hydroelectric power scheme present features that make it an interesting case study. Worthy of mention are the inclination and length of the unlined pressure tunnel, the high water head and the great depth of the powerhouse cavern. In projects of this type, the main effect of the internal water pressure in the pressure tunnel is the establishment of seepage from the tunnel into the rock mass, which can reach the adits and the powerhouse cavern. This seepage is influenced by several factors, such as the geometry of the underground openings, the rock mass properties-namely, the joints characteristics-and the stress state resulting from the excavation and from the internal water pressure. This article presents the main features of the underground structures of the Venda Nova II scheme and a detailed description of the observed behaviour during the first infilling of the pressure tunnel. A three-dimensional multi-laminated numerical model of the rock mass hydromechanical behaviour was developed to help understand the observed behaviour. The model assumptions in regard to the geometry of the openings, the jointing pattern, the rock mass hydraulic and mechanical behaviour, as well as the hydromechanical interaction, are described. Results obtained with the numerical model are presented and compared with the observed behaviour. Finally, the validity and importance of the numerical tools
\end{abstract}

L. N. Lamas $(\bowtie) \cdot$ N. S. Leitão

National Laboratory for Civil Engineering (LNEC),

Lisbon, Portugal

e-mail: 1lamas@1nec.pt

C. Esteves - N. Plasencia

Energies of Portugal (EDP), Porto, Portugal for the interpretation of the rock mass hydromechanical behaviour is discussed.

Keywords Unlined pressure tunnel · First infilling · Observed behaviour - Hydromechanical analysis

\section{List of Symbols}

$E, v \quad$ Young's modulus and Poisson's ratio

$K_{i j}^{s}, K_{i j}^{c}, K_{i j}^{e c} \quad$ Permeability tensor of a joint set, of the continuum and of the equivalent continuum

$K_{i}^{c}, K_{0}^{c} \quad$ Permeability of the continuum in a principal direction and its initial value

$g \quad$ Acceleration of gravity

$v_{\mathrm{p}} \quad \mathrm{p}$-Wave velocity

$\lambda \quad$ Joint frequency

$e, e_{0}, e_{\text {res }} \quad$ Joint aperture, initial joint aperture and residual joint aperture

$\omega \quad$ Kinematic viscosity of water

$\Delta \varepsilon \quad$ Strain increments

$\beta \quad$ Empirical hydromechanical parameter

$\sigma_{\mathrm{n}} \quad$ Normal stress

$k_{\mathrm{n}} \quad$ Normal stiffness

\section{Introduction}

A correct assessment of the performance of pressure tunnels and shafts of hydroelectric schemes is of considerable importance, owing to the high internal and external water pressures, to their length-which, in the case of pressure tunnels, may reach several kilometres-and to the geotechnical and hydrogeological conditions that may occur. The tendency to build underground hydraulic waterways, at increasing depths, is due to the increase of the efficiency of 
the excavation methods, to the smaller environmental impacts and to the advantages that it has from the mechanical and hydraulic points of view. Those considerations led to the optimisation of the Venda Nova II configuration (Alves et al. 1997).

The main purpose of the final lining of a pressure tunnel or shaft is to provide safe and economical operational conditions during its life span. This is achieved if the lining satisfies several requirements, the most important of which are related to the limitation of water losses, hydraulic jacking potential and frictional head losses, and to the stability of the waterway and adjacent slopes or underground openings.

Concrete linings are sometimes used to assure tunnel stability and hydraulic efficiency. However, they cannot be considered impervious because the tensile strains induced by cooling and shrinkage after concreting and by the internal water pressure exceed, by far, the admissible value for the concrete. Furthermore, a gap may form in the interface between concrete and rock, owing to difficulties in the concreting operation and contraction of the concrete caused by the circulation of water coming from the reservoir at a lower temperature. This gap means that no continuous contact between the lining and the rock exists. Therefore, even for low internal pressures, fissures are formed, and the confinement requirements against hydraulic jacking have to be satisfied.

Unlined sections may be used if some strict criteria are met. A fundamental criterion, which may exclude from the beginning consideration of an unlined tunnel, consists of the existence of a good quality rock mass, with adequate mechanical characteristics to assure the tunnel stability, and with low permeability in order to limit seepage.

Confinement criteria, which are dependent on the distribution of the water pressure and the state of stress in the rock mass, are important because they condition the alignment of the pressure tunnel. The existence of an adequate confinement is necessary in order to avoid the occurrence of hydraulic jacking and its associated consequences. Hydraulic jacking develops in a rock fracture when the water pressure exceeds the total normal stress, thus, increasing the fracture aperture and conductivity, which results in larger flow rates towards the rock mass. This phenomenon may induce high water pressures in distant zones, and has been responsible for several accidents in this type of tunnel (Lamas 1993). Besides, seepage from unlined pressure tunnels into the rock mass may result in operational, environmental or economic problems, such as leakage in nearby adits, the powerhouse cavern and even to the surface, changes in the rock mass water table or excessive water losses.

A third criterion that needs to be met in order to consider the unlined option is related to the higher frictional head losses due to the roughness of the rock mass surfaces, when compared with a much smoother cast concrete surface, and to the consequent need to increase the tunnel cross section area.

The rock mass mechanical and hydraulic behaviour over the several stages of life of a pressure tunnel is complex. The excavation of the tunnel, with or without the construction of a support system, is followed by the infilling with water at high pressure, and seepage into the rock mass depends on the confinement. Therefore, the models used for simulation of the processes involved should take into account the mechanical behaviour of the fractured rock mass and the support, flow characteristics and interaction between mechanical and hydraulic problems.

The main feature that influences the behaviour of the pressure tunnel of the Venda Nova II hydroelectric power scheme is that it is unlined along most of its length. Despite the high water head, this solution was possible due to the good quality of the rock mass from the mechanical and hydraulic points of view, and to the adequate confinement conferred by its location at great depth.

The first infilling of the Venda Nova II unlined pressure tunnel followed a detailed schedule. A monitoring plan was specifically prepared for this phase, which included the measurement of flow rates, water pressures and displacements inside the underground structures, surface observation in order to detect any major leakage, and monitoring water springs and boreholes water levels in order to control underground water. It was possible to monitor adequately the rock mass behaviour during the first infilling and to take the necessary measures to face the infiltrations that occurred in some adits and caverns due to leakage from the pressure tunnel, and, thus, to complete the infilling in a satisfactory fashion.

In order to help understand the observed rock mass behaviour during the first infilling, a three-dimensional, hydromechanical, multi-laminated numerical model was developed, which includes the terminal zone of the pressure tunnel, the powerhouse and neighbouring adits. The motivation for this numerical analysis of the water flow originated from the leakage that occurred into the adits near the pressure tunnel, while the powerhouse had, as expected, low infiltrations. Several situations were analysed in regards to the rock mass properties, the behaviour of the joints and the hydromechanical interaction mechanisms.

\section{The Venda Nova II Reversible Hydroelectric Scheme}

\subsection{General Information}

The Venda Nova hydroelectric power plant is located in the north-western region of Portugal, within the Cávado river 
mountainous basin (Fig. 1), the Portuguese region with the highest rainfall values (Oliveira et al. 2004).

The original power plant consists of a concrete dam in the Rabagão river, a tributary on the left bank of the Cávado river, that, through a hydraulic conduit of about $3.9 \mathrm{~km}$ in length, two-thirds in tunnel and one-third in penstock, feeds three turbines installed in the powerhouse building, delivering the turbined water back to the Cávado river upstream of the Rabagão confluence. By the time of its construction, in the 1950s, this scheme produced roughly one-quarter of the net consumption of electric energy in Portugal. After the construction of the other three developments in the same basin-Alto Rabagão, Paradela and Salamonde-the role of the Venda Nova development became fundamental in the management of the electric production of this system.

To increase the use of available resources in the reservoirs created by the dams of Venda Nova and Salamonde, Energies of Portugal (EDP) decided, in 1996, to repower the old scheme of Venda Nova. The large $420 \mathrm{~m}$ head created between these two reservoirs, achieved along a stretch of merely $4.5 \mathrm{~km}$, is unique in Portugal and has great energy production potential. This repowering project, Venda Nova II, was mostly built underground in the granite rock mass of the river Rabagão left bank. The construction involved several tunnels, $4.5 \mathrm{~km}$ of which are hydraulic unlined tunnels, several shafts and two main caverns for the powerhouse and the power transformers, located at depths of more than $320 \mathrm{~m}$. The underground powerhouse is equipped with two vertical-axis reversible units with a unit output of 97.1 MW/106.4 MVA and an average yearly power production of about $220 \mathrm{GWh}$. Each unit consists of a Francis pump turbine and a directly coupled synchronous motor-generator that rotates at a speed of $600 \mathrm{rpm}$.

The Venda Nova II scheme represents a very positive contribution towards increasing and improving the production efficiency of the Portuguese national electricity system, especially considering that, through a suitable management of water, it allows:

- Greater and better hydroelectric use of the hydraulic potential of the Rabagão river between the Venda Nova and Salamonde reservoirs, thus, decreasing discharges and concentrating the electricity production during shorter periods and periods of greater demand;

- Greater use in pump mode of excessive electric energy produced by the thermal plants at low consumption periods and greater availability in generator mode of energy and power in the periods when consumption significantly increases;

- By pumping, to improve the conditions in order to regulate the energy produced by wind, whose nature is intermittent.

\subsection{Layout}

The Venda Nova II scheme presents complex technical aspects associated with the design and performance of the different elements of construction. It is essentially composed by new hydraulic tunnels and a new powerhouse complex (Figs. 2, 3).

The Venda Nova II hydraulic system consists of:

- Upper intake, in the Venda Nova reservoir, with a shaft where the operation and safety elements are located;

- Lower intake, in the Salamonde reservoir;

- Unlined headrace tunnel, inclined at $15 \%$ and $2.8 \mathrm{~km}$ long, with a $6.3 \mathrm{~m}$ diameter modified circular section, establishing the connection between the upper intake and the powerhouse. The stretch upstream of the powerhouse is steel-lined and preceded by an upper sand trap;
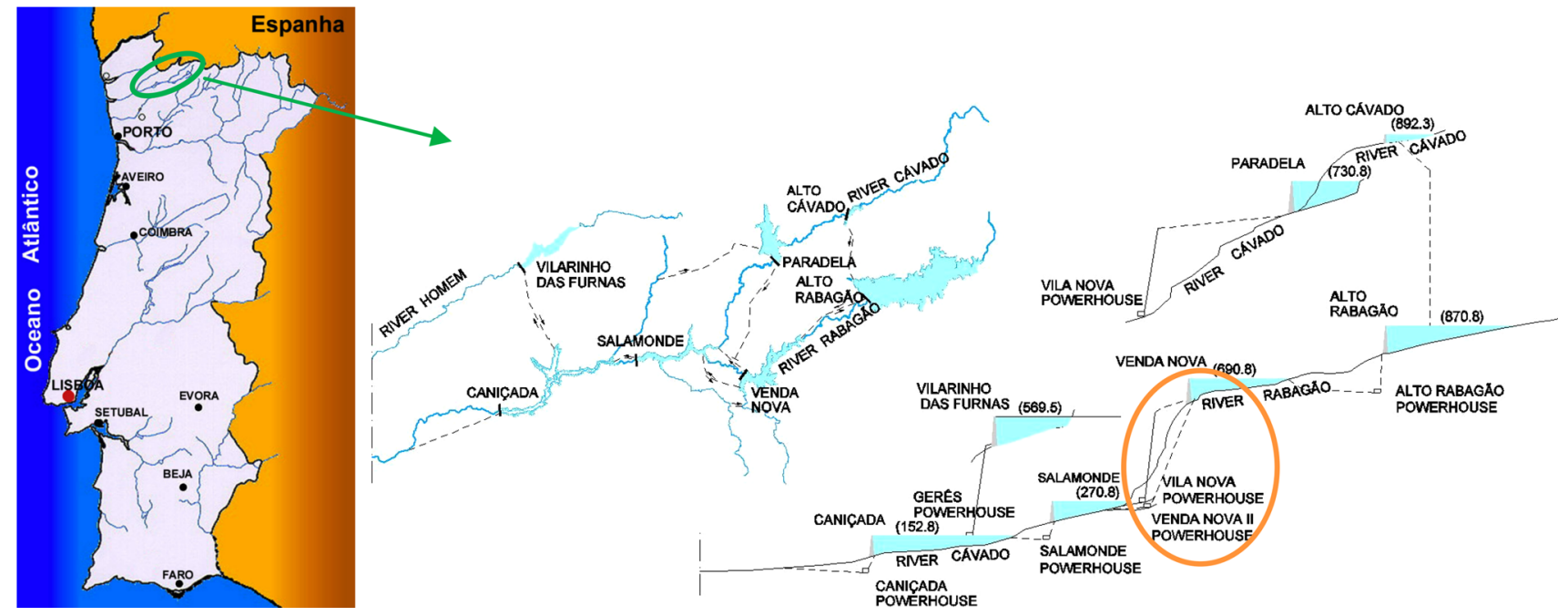

Fig. 1 Venda Nova II project location and the Cávado-Rabagão hydroelectric system 
Fig. 2 Powerhouse complex area

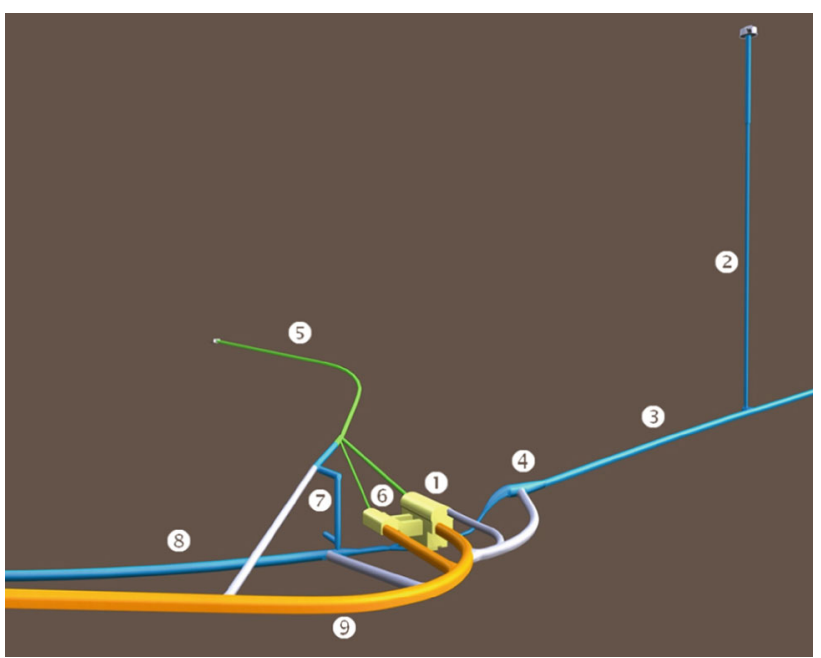

1 - Powerhouse cavern

2 - Upper surge shaft/tank

3 - Headrace tunnel

4 - Upper sand trap

5 - Ventilation gallery

6 - Transformers chamber

7 - Lower surge tank

8 - Tailrace tunnel

9 - Access tunnel
Fig. 3 Vertical and horizontal sections through the headrace tunnel upstream of the powerhouse
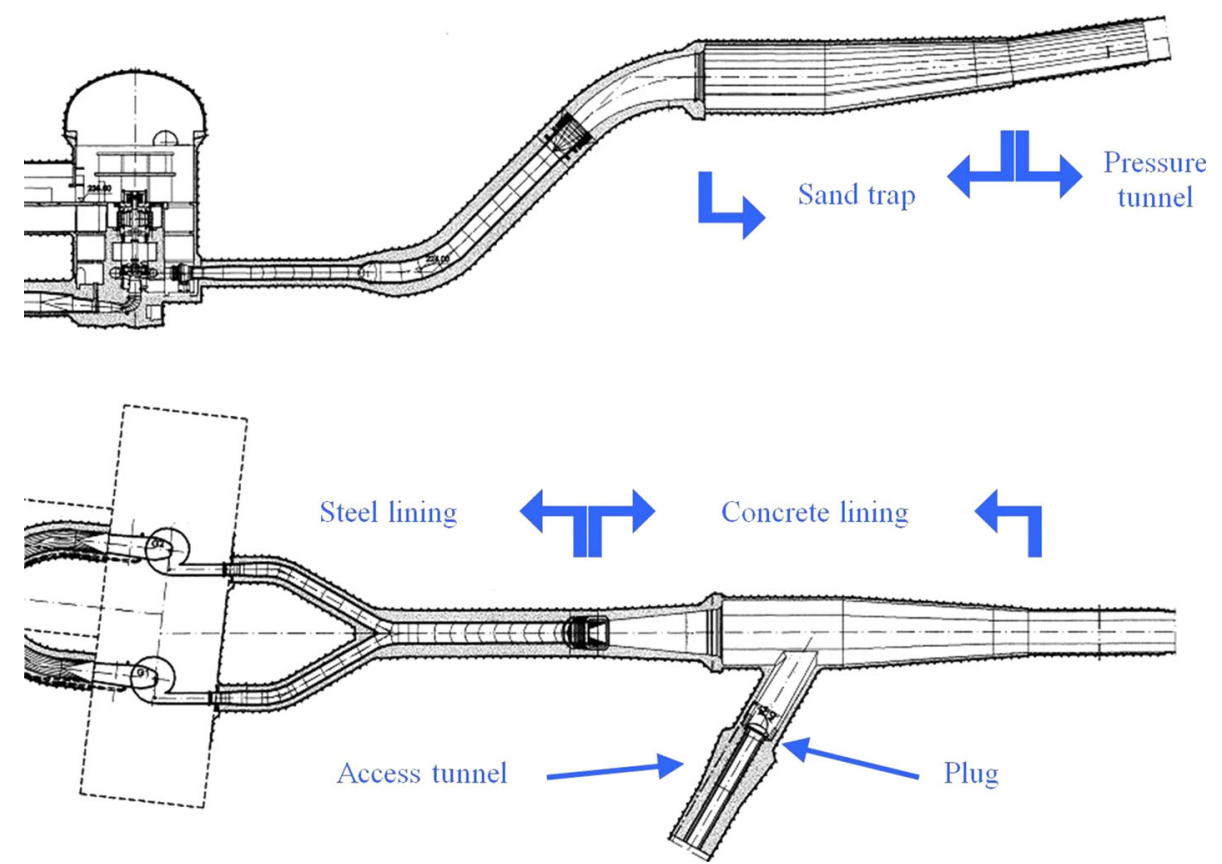

- Unlined tailrace tunnel running between the lower sand trap and the lower intake, sub-horizontal, $1.4 \mathrm{~km}$ long and with a $6.3 \mathrm{~m}$ diameter modified circular section;

- Upper surge tank, $500 \mathrm{~m}$ upstream of the powerhouse, consisting of an unlined, $4.5 \mathrm{~m}$ diameter vertical shaft, $420 \mathrm{~m}$ high, and an expansion reservoir at the surface;

- Lower surge tank, consisting of a concrete-lined, $5.0 \mathrm{~m}$ diameter vertical shaft, $58 \mathrm{~m}$ high, with two chambers;

- Two plugs equipped with seal doors, located in the adits used for construction of the headrace and tailrace tunnels.

The new powerhouse complex consists of:

- A powerhouse cavern, located in an intermediate position of the hydraulic tunnels, at a depth of about $350 \mathrm{~m}$, with an area of $20 \times 60 \mathrm{~m}^{2}$ on the plan and a maximum height of $40 \mathrm{~m}$, where the two reversible units are installed with their respective command and control equipment, as well as elements of hydraulic protection;

- An additional cavern and respective connecting tunnels, where two power transformers are installed;

- Ventilation and safety tunnels establishing a second connection from the two caverns to the surface;

- An unlined access tunnel with a diameter of $8 \mathrm{~m}$, inclined at $11 \%$ and $1.5 \mathrm{~km}$ long, also used for running the power and control cables from the power transformers to the surface support building;

- Switchgear and respective support building, located near the access tunnel entrance.

The positioning of the headrace tunnel at depth, as an alternative to the traditional sub-horizontal solution, was 
preferred in order to adopt an unlined solution, which requires adequate overburden, and also for hydrogeological reasons to protect the underground water (Alves et al. 1997). The adoption of a slope of $15 \%$ permitted the removal of debris from excavations using traditional methods. The slope of the tailrace tunnel resulted from the decision to empty it via the powerhouse.

To control the hydraulic transient regimes generated by the operation of the reversible units, associated to water hammer pressure variations and to the occurrence of speed rises and potential runaway of the units, two surge tanks were adopted, one upstream and another downstream of the powerhouse, thus, improving the system's operation in terms of speed regulation stability. From a strictly hydraulic point of view, their location should be as close as possible to the reversible units, although other factors also constrained their location.

Concerning the upper surge tank, the topographic level required for the expansion reservoir, and its location in an accessible area with no important geologic accidents, led to a location away from the powerhouse and to the construction of the $420 \mathrm{~m}$ high connection shaft. The location of the lower surge chamber was also determined by geotechnical reasons, by its incorporation into the complex scheme of access and auxiliary tunnels, and by the construction strategy in the powerhouse area.

\subsection{Rock Mass Properties}

The Venda Nova II excavations intersected mostly (92\%) hercynian granites (middle to coarse-grained porphyritic), but also $(7 \%)$ metamorphic rocks (hornfels and metagraywackes) and (1\%) Gerês granites (highly fractured and weathered) (Cotelo Neiva et al. 2000). The orientation of regional major faults is NE-SW and NW-SE, and also NNE-SSW to N-S. A very important fault was crossed by the tailrace tunnel. As usually expected for crystalline rocks, the permeability is very low. Nevertheless, high hydraulic conductivities were expected to be found in fault zones and near the surface. Figures 4 and 5 illustrate the geostructural conditions along the project and in the zone of the powerhouse caverns.

In the zone of the powerhouse, three major joint sets were identified in the granitic rock mass. Their main properties are indicated in Table 1 (Lima et al. 2002) following the International Society for Rock Mechanics (ISRM) methodology (ISRM 1978, 1981), and their orientations are represented in Fig. 6, where the axis of the powerhouse cavern is also indicated. Joint set F1 is the most representative one. There is a fourth joint set with orientation $\mathrm{N} 40^{\circ}-50^{\circ} \mathrm{E}$, sub-vertical, but it is not a predominant one.

Based on the site investigation and on a vast programme of field and in situ tests, including samples from the long

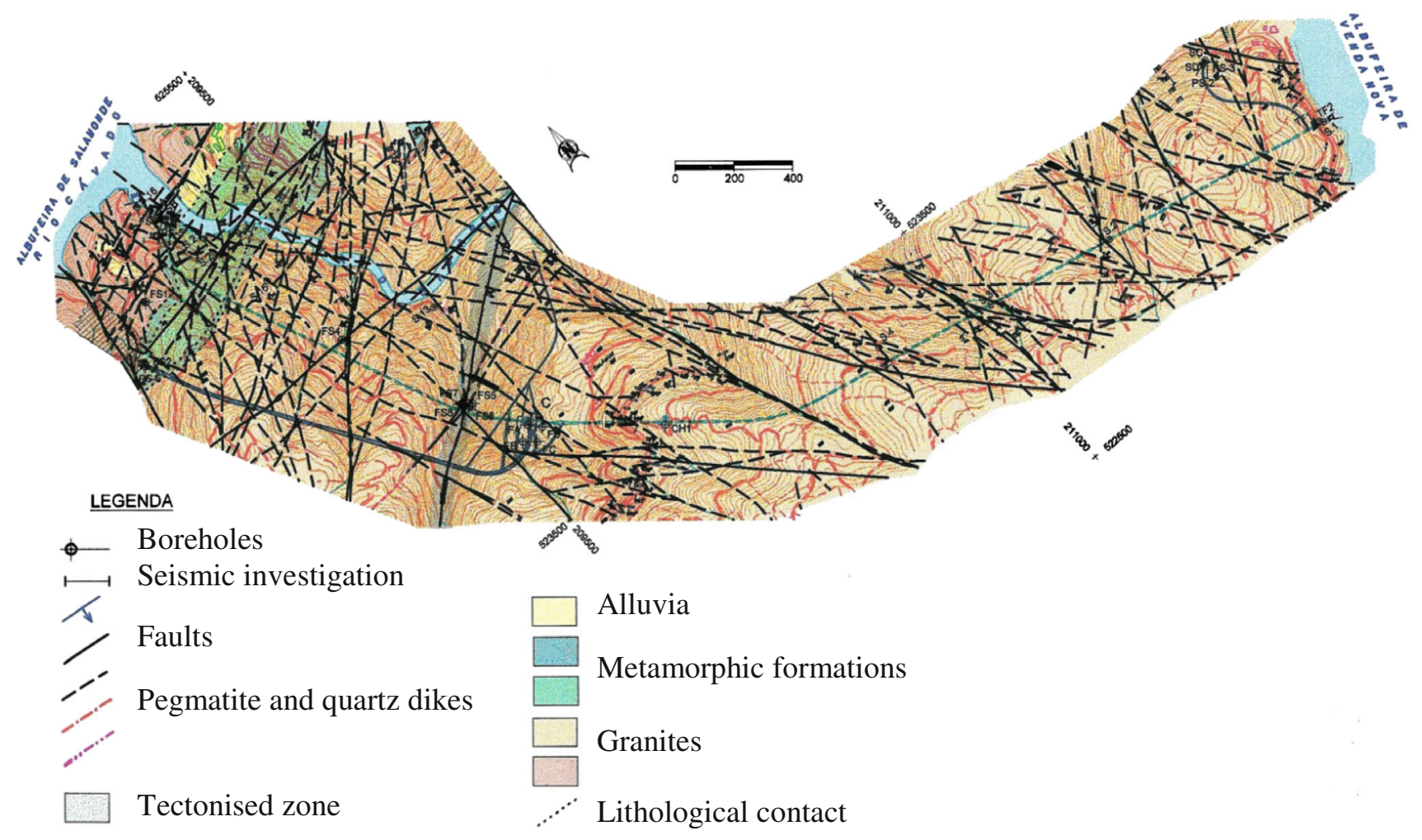

Fig. 4 Geostructural and lithological local conditions (Plasencia 2003) 
Fig. 5 Geostructural context near the powerhouse (Plasencia et al. 2000)

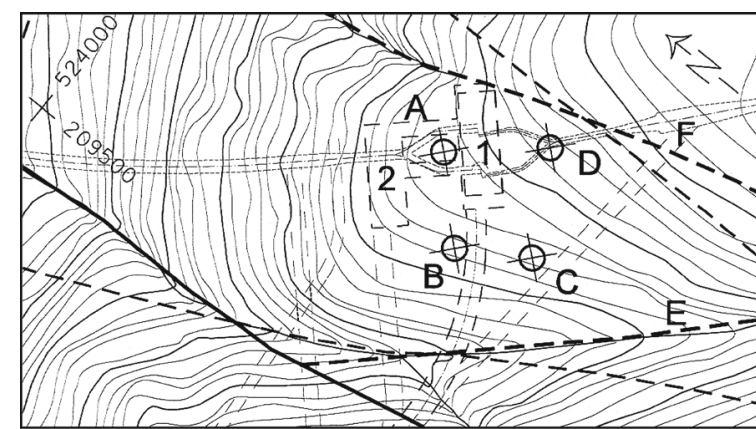

1 - powerhouse cavern

2 - transformers cavern

A, B, C, D - deep, vertical boreholes

E, F - major faults detected at the surface
Table 1 Joint set properties in the powerhouse zone

\begin{tabular}{llll}
\hline Joint set & $\mathrm{F} 1$ & $\mathrm{~F} 2$ & $\mathrm{~F} 3$ \\
\hline Direction & $\mathrm{NS}$ & $\mathrm{N} 94^{\circ} \mathrm{W}$ & $\mathrm{N} 57^{\circ} \mathrm{E}$ \\
Inclination & $85^{\circ} \mathrm{W}$ & $87^{\circ} \mathrm{S}$ & $13^{\circ} \mathrm{NW}$ \\
Persistence (m) & $3-10$ & $1-3$ & $1-10$ \\
Wall weathering & $\mathrm{W} 1-2$, occasionally W4 & $\mathrm{W} 1-2$, occasionally W3 & $\mathrm{W} 1-2$ \\
Aperture (mm) & Closed to 0.5, sometimes 2.5 & Closed to 0.5 & Closed to 0.5 \\
Filling (mm) & None to 2.5 & None to 0.5 & None to 0.5 \\
Roughness & Rough, plane, & Undulating, poorly & Undulating, poorly rough, \\
& sometimes polished & rough to rough & sometimes rough stepped \\
Seepage & Dry, occasionally with & Dry & Dry \\
& continuous water flow & & \\
Spacing (m) & 1-2 & 2-3, sometimes 5-6 & 2-3, sometimes 1 \\
\hline
\end{tabular}

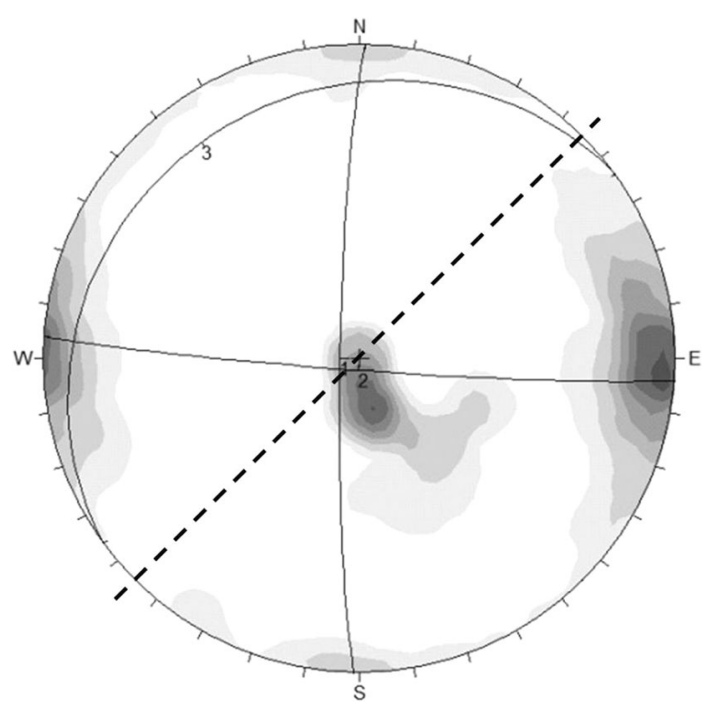

Fig. 6 Isodensity diagram, lower hemisphere projection. The dashed line represents the cavern axis

borehole of the powerhouse zone, the rock mass along the hydraulic layout was classified into three distinct geotechnical zones, with the properties presented in Table 2, where $v_{\mathrm{p}}$ is the p-wave velocity, $E$ is the Young's modulus and UCS is the uniaxial compressive strength measured in the rock cores.

As a confirmation of the good rock mass quality, approximately $75 \%$ of the hydraulic tunnels were excavated in the higher quality geotechnical zone (ZG1 and ZG2). The intermediate and lower quality geotechnical zones were concentrated in the superficial regions, close to the water intakes and the upper part of the upstream surge chamber, as well as in regions where faults zones were intersected (Fig. 7).

The geotechnical characteristics of the rock mass were a decisive factor for selecting the location of the powerhouse caverns, looking for good overburden conditions as well as compatibility with the most important geologic formations, in accordance to the required submergence of the reversible units. Once the approximate powerhouse location was selected, four vertical boreholes of about $350 \mathrm{~m}$ each were drilled from the surface and Lugeon tests were carried out inside them. The cross-hole seismic tomography helped to define the geological conditions (Plasencia et al. 2000). Figure 8 shows the locations of these boreholes and the boundaries between geotechnical zones.

From the powerhouse access tunnel, and crossing longitudinally the site of the main cavern, a survey adit was excavated, where large-scale field tests were performed to evaluate the rock mass deformability, and overcoring tests were carried out to assess the in situ state of stress.

The results of the overcoring tests carried out prior to the powerhouse construction and the back-analyses performed with the values of the displacements measured during the excavation phases led to the conclusion that the 
Table 2 Geological and geotechnical zoning of the rock mass along the hydraulic layout

\begin{tabular}{|c|c|c|c|c|c|c|c|c|c|}
\hline \multirow[t]{2}{*}{ Zone } & \multirow{2}{*}{$\begin{array}{l}\text { Degree of weathering } \\
\text { and of fracturing }\end{array}$} & \multirow[t]{2}{*}{ RQD } & \multirow[t]{2}{*}{$v_{\mathrm{p}}(\mathrm{m} / \mathrm{s})$} & \multirow[t]{2}{*}{$E(\mathrm{GPa})$} & \multirow[t]{2}{*}{ UCS (MPa) } & \multicolumn{4}{|c|}{ Permeability (Lugeon units) } \\
\hline & & & & & & $>10(\%)$ & $10-2(\%)$ & $2-1(\%)$ & $<1(\%)$ \\
\hline ZG3 & $\mathrm{W} 5, \mathrm{~F} 5$ to $\mathrm{W} 2-\mathrm{W} 3, \mathrm{~F} 4$ & $0-70$ & $800-2,800$ & $<20$ & $<30$ & 36 & 29 & 9 & 26 \\
\hline $\mathrm{ZG} 2$ & W2-W3, F3-F4 to W2, F1-2 & $70-95$ & $3,000-4,600$ & $20-40$ & $70-100$ & 1 & 27 & 9 & 63 \\
\hline ZG1 & $\mathrm{W} 1-2, \mathrm{~F} 2-\mathrm{F} 3$ to $\mathrm{W} 1, \mathrm{~F} 1$ & $95-100$ & $>4,800$ & $40-70$ & 110 & 2 & 2 & 4 & 92 \\
\hline
\end{tabular}

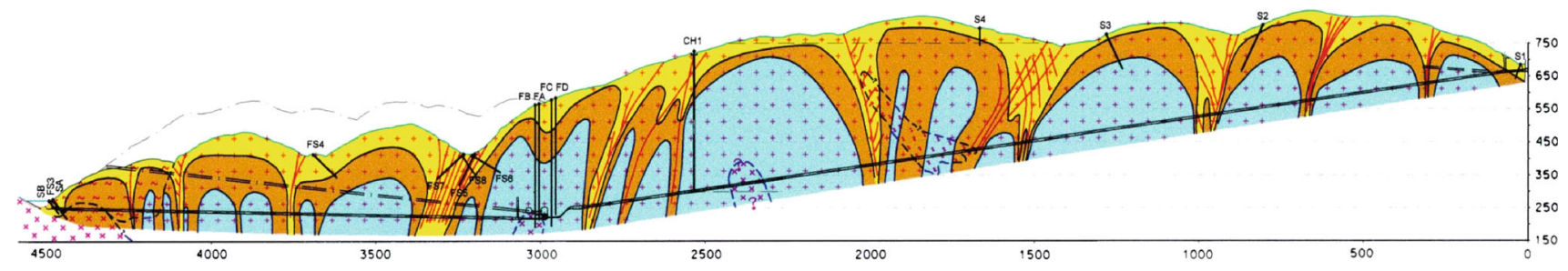

\section{LEGEND}

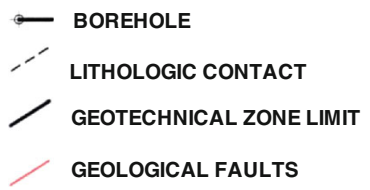

\begin{tabular}{|c|c|c|c|}
\hline & ZG1 or ZG2 & ZG3 & $\begin{array}{c}\text { FAULT OR } \\
\text { WEATHERING ZONE }\end{array}$ \\
\hline $\begin{array}{l}\text { BORRALHA } \\
\text { GRANITE }\end{array}$ & 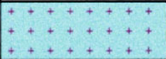 & 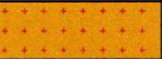 & 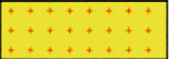 \\
\hline $\begin{array}{c}\text { ROSE } \\
\text { GRANITE }\end{array}$ & $\begin{array}{l}x \times x \times x \times x \\
\times x \times x \times x \times x \\
x \times x \times x \times x \times x\end{array}$ & $\begin{array}{r}x \times x \times x \times x \\
x \times x \times x \times x \\
x \times x \times x \times x \times\end{array}$ & 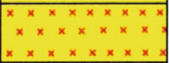 \\
\hline MIGMATITE & 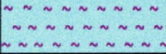 & & 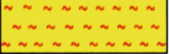 \\
\hline
\end{tabular}

Fig. 7 Longitudinal profile with the geotechnical zoning (Plasencia 2003)

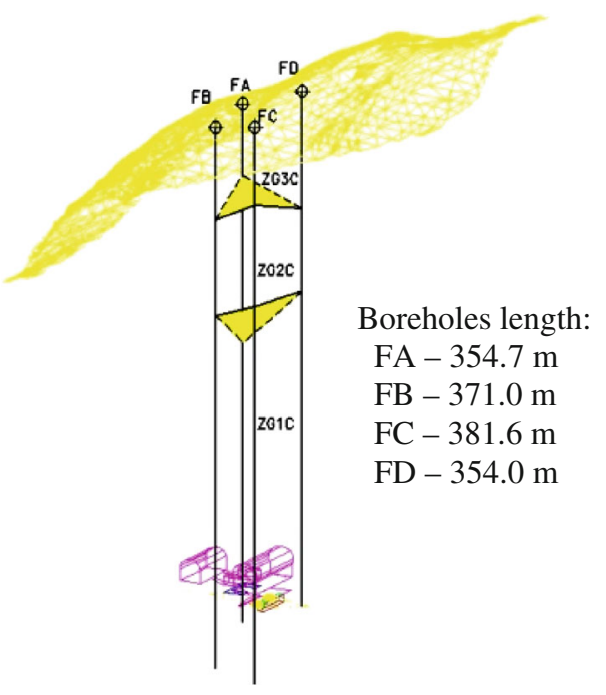

Fig. 8 Deep boreholes in the powerhouse rock mass and geotechnical zoning (Plasencia 2003)

vertical stress in the powerhouse area was nearly equal to the weight of the overburden, and the horizontal stress normal to the powerhouse axis is about 2.5 times higher than the vertical stress (Laboratório Nacional de Engenharia Civil; LNEC 2003a).

The photographs of Fig. 9 show aspects of the powerhouse and of the headrace tunnel, where the good rock mass quality can be confirmed.

\subsection{Main Actions Considered in the Design}

During the infilling of a pressure tunnel, the internal water pressure is the main action that must be taken into account. In the case of Venda Nova II, the internal water pressure is considerably high. Since the tunnel does not have a waterproofing element, it is assumed that water flow takes place, from and into the rock mass. The question that arises is to prevent possible situations of instability of the rock mass and uncontrolled water leakage problems caused by the hydraulic jacking of the rock mass. This can be achieved through appropriate confinement conditions, which include ensuring that, at any point of the rock mass, the water pressure is lower than the minimum principal stress. In the design of these pressure tunnels, it is usually considered acceptable that the internal pressure does not exceed the in situ stresses prior to tunnel excavation. In the design of the Venda Nova II hydraulic tunnels, the confinement criterion that was considered conditioned the choice of the tunnel alignment, ensuring that, at any point of the tunnel, the vertical stress due to overburden is higher than the internal water pressure.

In sections lined with conventional concrete, the predominant action is also the internal water pressure. Concrete linings considerably reduce the exchange of water with the rock mass, but they cannot be considered impervious because it is virtually inevitable that the concrete 

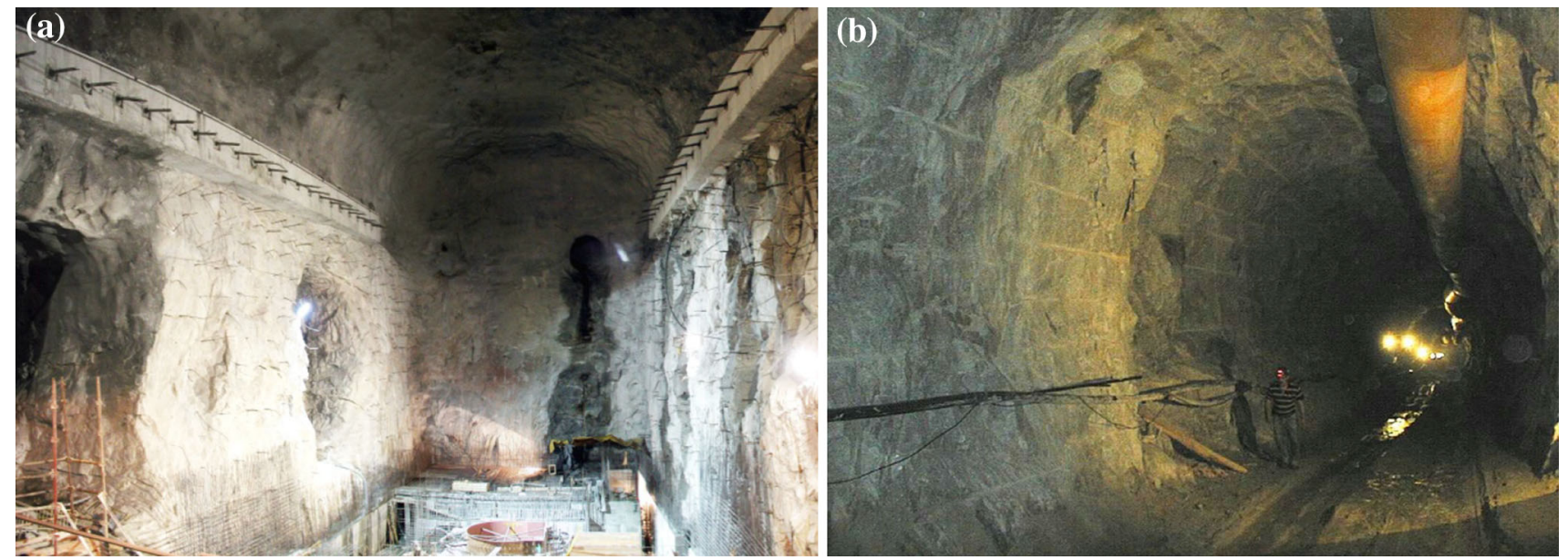

Fig. 9 Aspects of the good quality rock mass of the powerhouse (a) and headrace tunnel (b) (Plasencia 2008)

cracks under the action of internal pressure and of temperature variations imposed by water flow. Another action that can be important in concrete-lined sections is the external water pressure in the rock mass. In Venda Nova II, radial drains were provided in order to release the external pressure during emptying of the tunnels.

In the steel-lined sections, near the powerhouse, in addition to internal pressure and temperature variations, the external water pressure assumes a very important role in the design of the lining for situations of an empty tunnel.

The main effects of the actions consist of water percolation from the pressurised tunnels into the rock mass, and from the rock mass into the powerhouse cavern and the adits. Thus, the observation system to be implemented should pay special attention to the measurement of water pressures in the rock mass, water infiltrations and groundwater levels.

\subsection{Linings Used in the Hydraulic Tunnels}

In the design of the underground works, the traditional rigid solutions of reinforced concrete- "lined" solutionsusually adopted in Portuguese hydroelectric schemes were abandoned and flexible support structures with fibre shotcrete and rock bolting- "unlined" solutions-were used (Hidrorumo 1997). In fact, the rock mass also performs a structural function and the walls' roughness increase was balanced by the diameter enlargement. These solutions were associated with specific design criteria that need to be fulfilled: (a) to guarantee low flow velocities $(1.0-1.5 \mathrm{~m} / \mathrm{s})$; (b) to allow the fall of some rock blocks of small size; (c) to avoid the emptying of the system. The units are protected by racks placed in the sand traps.

According to the quality of the rock mass, four "unlined" typical sections were defined, with different rock bolts and shotcrete specifications, as well as a reinforced cast-in-place concrete-lined section. The total length of the rigid lining was around $7 \%$ in the headrace tunnel and $14 \%$ in the tailrace tunnel, in the locations shown in Fig. 10. As mentioned in the previous section, in the reinforced concrete-lined section, radial drains were provided in order to release the external pressure.

In order to minimise leakage, the headrace pressure tunnel near the powerhouse caverns complex has a reinforced concrete lining and the stretch upstream of the powerhouse is partially steel lined (see Fig. 3), complemented by a grout curtain, due to the high installed pressures (dynamic hydraulic heads of about $550 \mathrm{~m}$ ). A special treatment using injection with cement grout and synthetic resins was performed, mainly in the upper plug zone, located close to the intersection between the access adit and the headrace tunnel.

\section{First Infilling of the Headrace Pressure Tunnel}

\subsection{Procedures and Monitoring}

In unlined hydraulic tunnels, the first infilling is a very important phase. The rock mass hydraulic conditions are changed from the existing situation after the excavation period to the final design situation, where the total water pressures are installed. This process results in the filling of the fissures and voids in the rock mass and may induce leakage into the caverns and adits, and also to the surface. The filling velocity must be defined considering the geological and hydraulic conditions, in order to avoid any damage, mainly around the powerhouse and in the upper plug area (connection of adit G4 with the pressure tunnel).

Prior to the infilling of the hydraulic tunnels, the identification of all leakage points in the tunnels and caverns was carried out, with evaluation and registration of the flow 


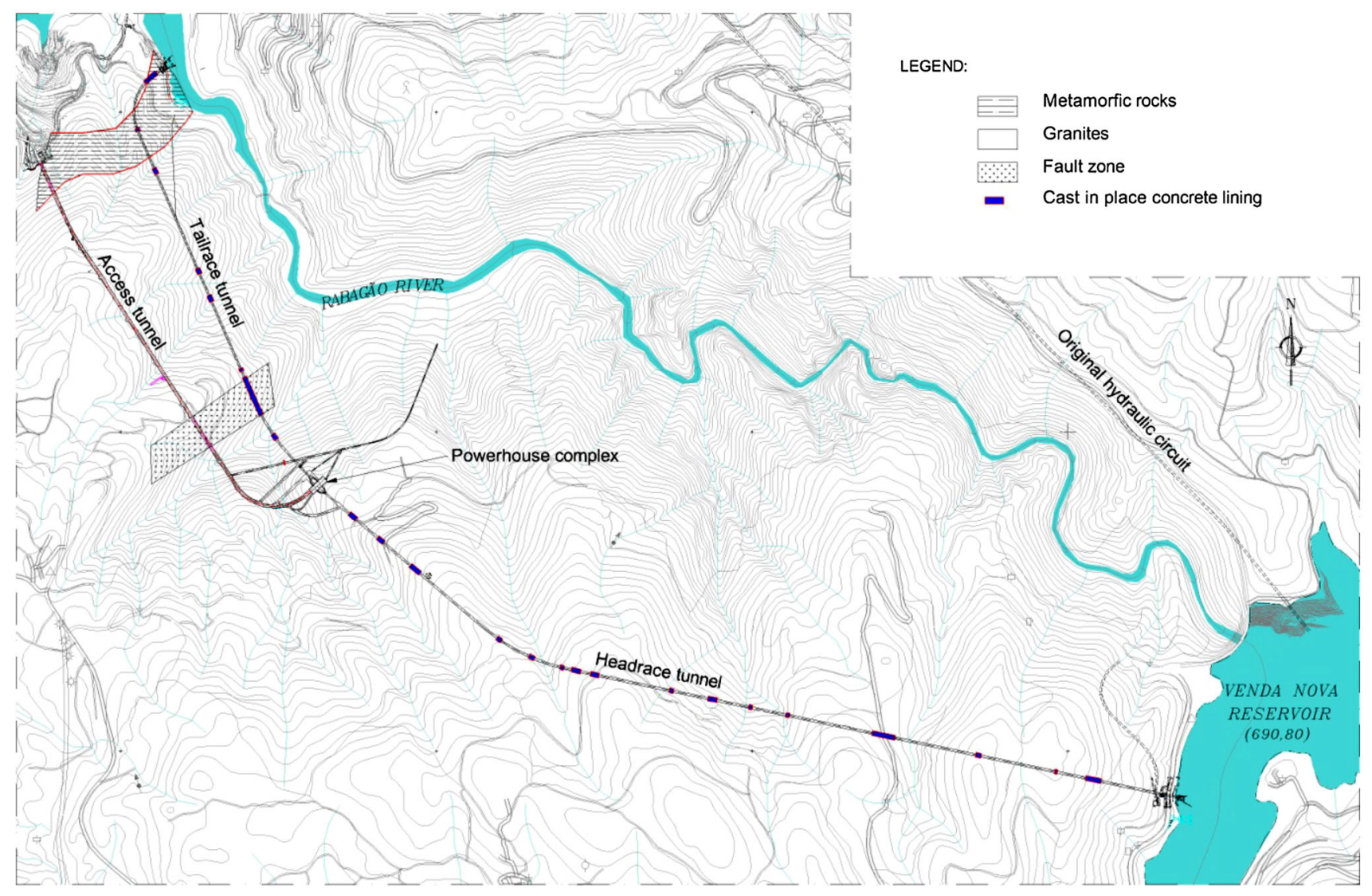

Fig. 10 Cast-in-place concrete lining applied in the headrace and tailrace tunnels

rates in the most important points. Surface water conditions were analysed by surveying some selected water springs and borehole water levels located at the surface above the tunnels (before, during and after construction, as well during the infilling).

A monitoring plan was established for the zone upstream of the powerhouse, which defined the monitoring system and the measurement frequencies (LNEC 2003b), and included:

- Piezometers in the powerhouse upstream and downstream walls, as well as in the upper plug;

- A large number of points of measurement of infiltrated flow rates in the adits and the powerhouse.

This complemented the existing monitoring system of the powerhouse, which includes rod extensometers mounted in two instrumented sections, thermometers, load cells in the anchors and systematic visual inspection of all the powerhouse walls. A high-precision digital manometer was installed in the penstock, close to the protection valve in the powerhouse, in order to measure accurately the water level in the tunnel.

In order to control the infilling operation, a specific "First Infilling Procedure" was defined, including responsibility assignment, monitoring system definition and schedule of water rises and monitoring steps. At each step, the filling operation was stopped for a period long enough to control the water level in the tunnels and to quantify the amount of leakage out of the headrace tunnel. This leakage was evaluated by the water level variation inside the tunnel, and the infiltrations into the underground facilities were continuously controlled, during the infilling periods and in the monitoring stages. When the leakage out of the tunnel was considered to be acceptable, the surface observations indicated the non-existence of flow and the leakage rate reduced with time, the infilling was continued.

\subsection{Observed Behaviour}

The infilling was scheduled to start from the tailrace tunnel, which is shorter than the headrace tunnel and has a much lower final water pressure, in one step. Filling of the headrace tunnel was done through controlled periodic opening of the intake gate, with durations that were adjusted by controlling the effective rate of water level increase inside the tunnel (about $10 \mathrm{~m} / \mathrm{h}$ in the first $250 \mathrm{~m}$ and a reduced rate of $5 \mathrm{~m} / \mathrm{h}$ in the last $150 \mathrm{~m}$ ). Monitoring stages were initially established at approximated every 
$100 \mathrm{~m}$ and later adjusted, depending on the behaviour of the measured leakage. Figure 11 represents the stages that were initially scheduled and the water level that represents the procedure that was actually followed. The drop in the water level, which was recorded during the stages at different pressures, represents the leakage from the headrace tunnel into the rock mass.

A large number of measurements were taken in order to control the safety of the underground structures and the operating conditions of the headrace pressure tunnel. Of particular relevance are the total values of the water infiltrations in the underground openings in the vicinity of the pressure tunnel. Figure 11 shows the measured infiltrations into the powerhouse and in adits G4 and G5, during the whole process of the first infilling, until they reached stability. Smaller infiltrations occurred in other tunnels, but they were not significant and were easily controlled and drained.

An important conclusion from Fig. 11 is that infiltration into the powerhouse was very low and was easily controlled by drainage. Besides, the four piezometers installed in the upstream wall of the powerhouse measured relatively low pressures, with a maximum value of $0.35 \mathrm{MPa}$ in one of them, while the others did not exceed 0.20 MPa. As expected, measurements of the rod extensometers did not change. Therefore, safety and operational conditions of the powerhouse were always guaranteed.

Infiltration into adits G4 and G5 required particular attention during the whole infilling process. In the first stages, the values of the water infiltrations into G4 were larger than those into G5. The water flowed into G4 mainly through a number of large conducting discontinuities with the orientation represented in Fig. 12a, which intersected the main joint set with orientation NNW-SSE to NS. In order to control the water inflow, it was decided to grout some of these discontinuities around $\mathrm{G} 4$, and to drill drainage holes in $\mathrm{G} 4$, in order to release the water pressure (Fig. 12b). This resulted in the expected decrease in the water infiltration into G4, but in an increase in the infiltration into G5. The effect of main drain, which, in the beginning was being performed by G4, was partially transferred to G5 due to the barriers created to the inflow into $\mathrm{G} 4$ by grouting. This can be clearly seen in the values of the infiltration into G4 and into G5 shown in Fig. 11, where the total infiltration into G4 plus G5 is also represented, and is a better measure of the observed infiltrations.

Monitoring of the surface water conditions during construction and after infilling indicated localised impact on the water table during construction and a recovery some time after the infilling. During the infilling phase, the observation of the surface conditions and monitoring of the water table showed no impacts on it.

\section{Numerical Model for Simulation of the First Infilling}

\subsection{General}

The safety and serviceability of pressure tunnels are conditioned by different aspects during their lifetime. During construction, the most important action is the release of the in situ state of stress due to excavation, but, in some cases, water pressures may also have to be considered (Lamas and Sousa 1993). Safety control is usually based on displacement analyses and on water inflow. In most cases, simple two-dimensional models are sufficient for a timely and adequate interpretation of the observed behaviour.
Fig. 11 First infilling: infiltration into the powerhouse and into adits G4 and G5 (colour figure online)

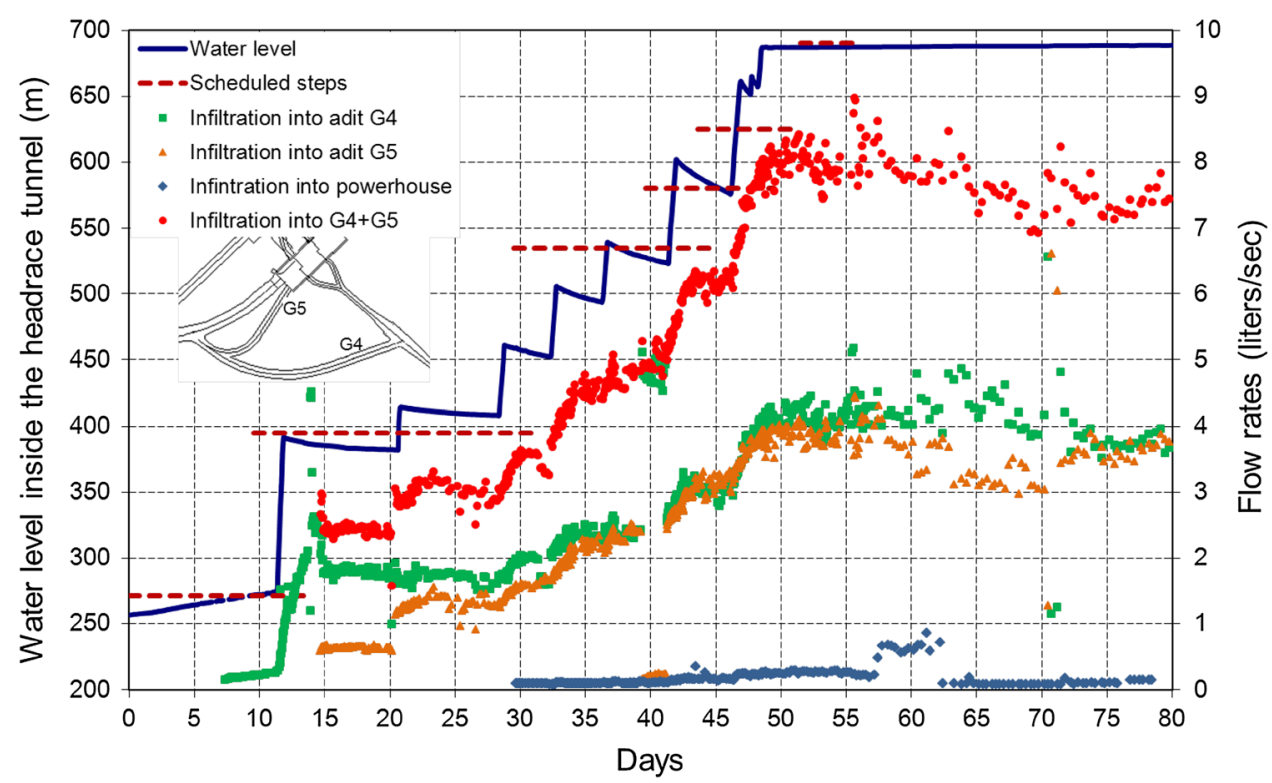



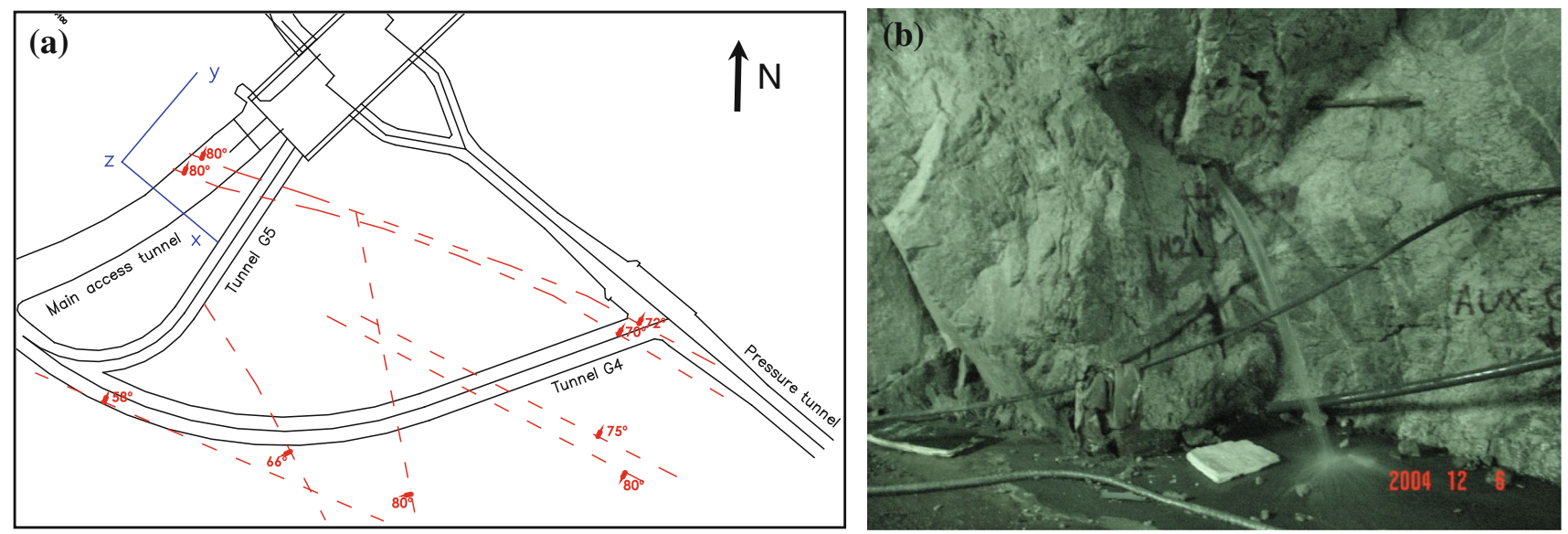

Fig. 12 Main conductive discontinuities (a) and large water flow collected by a drain hole located in adit G5 (b)

Before the first infilling starts, the rock mass is stabilised and all the required linings are in place. During the early stages of the infilling, still at low water heads, the main action to consider is the temperature change caused by the presence of water coming from the reservoir, usually at a lower temperature, which may have an important effect on the behaviour of the lining. In unlined tunnels, as the water head increases, the internal water pressure and the seepage from the tunnel to the rock mass become the most important actions.

The purpose of the instrumentation and monitoring of underground structures is to assess the safety and serviceability conditions, by comparing the collected data with the expected behaviour. In order to reach this objective, it is necessary to develop conceptual models which correspond to certain idealizations of the physical problem. The conceptual models shall be able to simulate, in each situation, the relevant aspects of the structure's behaviour. When the collected data deviate from the expected behaviour or design assumptions, the conceptual model must be improved to match as closely as possible the actual situation.

The first infilling of Venda Nova II is a well-documented case study in an extensively characterised rock mass, where a systematic monitoring system was implemented, which supplied a wealth of results. The measured flow rates revealed that relatively high leakage occurred into adits G4 and G5 near the pressure tunnel, and this was discussed in detail in the previous section. These two facts constituted the motivation for a deeper study of the hydraulic behaviour of the rock mass during the first infilling, which was performed after the scheme was in normal operation.

Keeping in mind the unlined nature of the pressure tunnel, the high water pressures and in situ stresses, and the influence that interaction between the mechanical and the hydraulic problems may have on the rock mass behaviour, a hydromechanical numerical model was developed. In this study, special effort was directed to revealing the influence of the presence of several, nearly parallel, large conducting discontinuities.

The simplifying assumptions that were adopted and the resulting conceptual model are described in detail in the next section, and this is followed by the presentation of its numerical implementation.

\subsection{Conceptual Model}

\subsubsection{Geometry}

For the study of this kind of problem in excavations with complex geometry, such as in the present case, it is necessary that the model represents the hydraulic tunnels as well as the other tunnels and caverns that are in their vicinity. Hence, a three-dimensional representation of the geometry of the excavations is necessary.

The underground structures represented in the Venda Nova II model are those deemed to be of greater influence for analysis of the phenomena that are to be simulated. Besides the headrace pressure tunnel, the powerhouse cavern, the main access tunnel and the adits identified as G4 and G5 were also included (Leitão et al. 2005). The geometry of the steel-lined section (see Fig. 3), where no flow exists, was represented in a simplified way and corresponds to the inclined and the lower stretches of the pressure tunnel. Figure 13 shows the three-dimensional domain of the model and the underground structures that were considered.

\subsubsection{Mechanical Behaviour}

The choice of the approach to consider-continuous or discontinuous-depends on the characteristics of each specific situation, namely, the rock mass jointing system, 


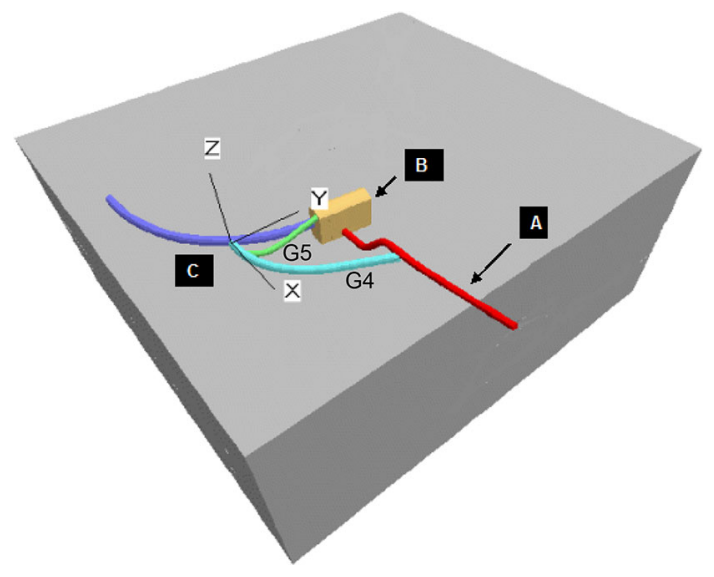

Fig. 13 Schematics of the headrace pressure tunnel $(A)$, main powerhouse cavern $(B)$ and adits $(C)$

the size of the excavations and the expected influence of the discontinuities on the global behaviour of the rock mass.

The rock mass of the Venda Nova II hydraulic tunnels has very high mechanical properties. At the depth of the powerhouse, the stresses are high and the discontinuities can be considered closed from the mechanical point of view. It can be assumed that the mechanical behaviour of the rock mass is not significantly influenced by the discontinuities, and, therefore, consideration of a continuous medium approach is adequate. Thus, the granitic rock mass in the zone of interest was simulated as a linear elastic, isotropic, homogeneous, continuous medium.

\subsubsection{Hydraulic Behaviour}

The rock mass hydraulic behaviour is governed by the jointing pattern, because seepage occurs through the discontinuities' network. A continuous approach may be acceptable for the hydraulic behaviour, but the rock mass hydraulic properties must take into account the influence of the discontinuities.

An objective of the current numerical simulations consists in assessing the role of several, nearly parallel, large conducting discontinuities on the hydraulic behaviour of the rock mass. In order to enable this, the rock mass is simulated as an equivalent continuum, using the concept of a multi-laminate medium (Zienkiewicz and Pande 1977), which integrates the hydraulic properties of the rock material with the geometric and hydraulic properties of the discontinuities, grouped in joint sets (Lamas 1993). Laminar flow and steady-state conditions are assumed in the simulation of the unconfined water flow.

In a general multi-laminate approach, the rock mass permeability tensor, $K_{i j}^{e c}$, can be decomposed into two components:
$K_{i j}^{e c}=K_{i j}^{c}+\sum_{s=1}^{n} K_{i j}^{s} \quad$ with $\quad K_{i j}^{s}=\frac{g \lambda e^{3}}{12 v_{\omega}} \quad(i, j=1,2,3)$

$K_{i j}^{c}$ is the rock mass permeability tensor without the consideration of the discontinuities. It is usually considered to be isotropic for the conditions prior to the excavation of the underground structures. $K_{i j}^{s}$ is the permeability tensor relative to the contribution of each of the $n$ joint sets. In this equation (known as the cubic law for laminar flow), $g$ is the acceleration of gravity, $\lambda$ the joint frequency, $e$ the joint aperture and $v_{\omega}$ the kinematic viscosity of water. Thus, by using Eq. (1), once the geometric properties of the main joint sets are known, it is possible to calculate the anisotropic permeability tensor of the rock mass.

\subsubsection{Hydromechanical Behaviour}

For modelling the hydromechanical behaviour of rock masses, it should be borne in mind that the rock mass permeability does not present a constant value. Seepage takes place through the discontinuity network and the permeability of a joint is a function of the cube of its aperture. Therefore, the rock mass permeability depends on the aperture of the joints and, consequently, on the normal stresses at the joints. This is why, in an equivalent continuous-medium approach, a relationship between the rock mass permeability and the state of strain is sometimes used.

On the other hand, the water flow generates seepage forces, which are mechanical loadings that must also be taken into account. In turn, the seepage forces also introduce a change in the stress field and, consequently, in the joints' aperture and in the rock mass permeability. Whenever this hydromechanical interaction is deemed to be relevant for the simulation of the rock mass behaviour, it shall be considered in the model and studied as an integrated process. This is often the case in the analysis of unlined pressure tunnels under high internal water pressure.

A review of hydromechanical couplings in fractured rock, with special emphasis on hydromechanical interactions as a result of human activities such as underground injection and underground construction, can be seen in Rutqvist and Stephansson (2003).

For simulation of the hydromechanical behaviour of the Venda Nova II underground structures, the component $K_{i j}^{c}$ of the permeability tensor given in Eq. (1) is calculated using a law proposed by Wei (1992), according to which permeability decreases when compression strains increases, and vice versa. The permeability in a principal direction $K_{i}^{c}$ is a function of an initial value $K_{0}^{c}$ and of the sum of the strain increments $\Delta \varepsilon$ in the orthogonal plane, where $\beta$ is an empirical parameter: 


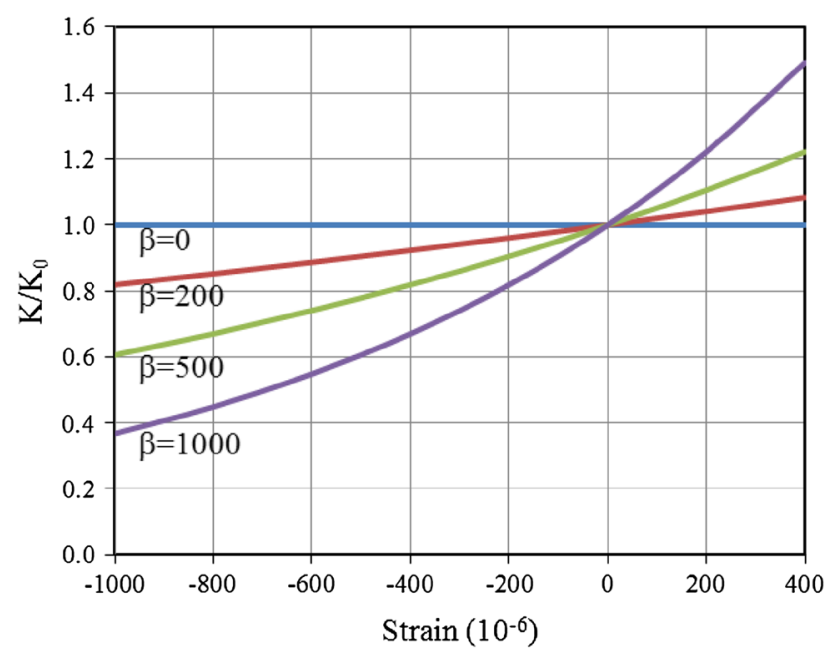

Fig. 14 Influence of strain in the permeability of a continuous medium

$K_{i}^{c}=K_{0}^{c} e^{-\beta\left(\Delta \varepsilon_{j}+\Delta \varepsilon_{k}\right)} \quad(i=1,2,3 ; j=2,3,1 ; k=3,1,2)$

Figure 14 shows the permeability variation with strain according to this law for several values of $\beta$.

For calculation of the component $K_{i j}^{s}$ of the permeability tensor given in Eq. (1), the apertures $e$ of the joint sets are calculated considering an initial value $e_{0}$ and the stress component $\sigma_{\mathrm{n}}$, normal to the joint set, using a constant normal stiffness $k_{\mathrm{n}}$, and an irreducible residual aperture $e_{\text {res }}$ (see Fig. 15):

$e=e_{0}-\frac{\sigma_{\mathrm{n}}}{k_{\mathrm{n}}} \quad$ with $\quad e \geq e_{\text {res }}$

The rock mass permeability tensor, $K_{i j}^{e c}$, obtained in this way, embodies the influence of the stress-strain behaviour on the hydraulic behaviour. For consideration of the influence of the water flow on the mechanical behaviour, the seepage forces are calculated and are input as loads.

\subsection{Numerical Implementation}

\subsubsection{Hydromechanical Coupling Procedure}

The equivalent continuous model described above allows the use of a continuum-based modelling package. For this analysis, the FLAC ${ }^{3 \mathrm{D}}$ (Itasca Consulting Group 2002) code was adopted. FLAC ${ }^{3 \mathrm{D}}$ is a widely used commercial code that is designed for rock and soil mechanics, and can also handle hydraulic and fully coupled hydromechanical processes.

For the proposed hydromechanical model, based on an iteratively coupled approach, the FLAC ${ }^{3 \mathrm{D}}$ code is executed sequentially to model the mechanical and hydraulic behaviour. Although the FISH programming language

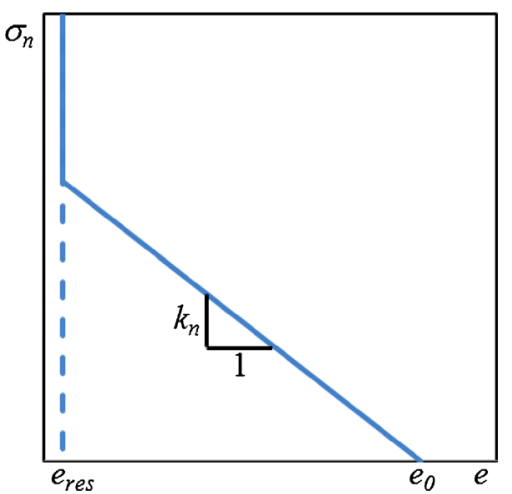

Fig. 15 Normal stress versus joint aperture

embedded within FLAC ${ }^{3 \mathrm{D}}$ enables the computation of the strain-induced changes in permeability, it was decided to perform the computation externally using a FORTRAN program (Leitão and Lamas 2006a). The structure chart in Fig. 16 illustrates the main sequence of calculations for this program.

First, the program reads the data describing the joint sets and the hydraulic properties. Then, the initial mechanical conditions corresponding to a state of equilibrium and the initial pore pressure distribution are computed before the coupled analysis is started. For all iterations, the new permeability coefficients are calculated. After solving the flow-only and mechanical-only calculations, the convergence criteria in potentials and displacements are verified. If convergence is not reached, a new iteration takes place.

The information between the FLAC ${ }^{3 \mathrm{D}}$ and the FORTRAN codes is passed using ASCII files, which can be read or written by FISH subroutines during FLAC ${ }^{3 \mathrm{D}}$ execution. The set of commands which control the running of FLAC $^{3 D}$ is given through the "FLAC3D.INI" initialisation program, which is automatically accessed upon starting FLAC $^{3 \mathrm{D}}$ with a run-time FORTRAN function.

\subsubsection{Finite Difference Grid}

To study the hydromechanical behaviour of the Venda Nova II scheme, a segment of the pressure tunnel, three access tunnels and the main powerhouse cavern were embedded into a $391 \times 438 \times 170 \mathrm{~m}^{3}$ rock block (Fig. 13).

The mesh was built using only brick primitives. At first, the excavation was created using FISH functions to move the locations of the grid points for each primitive to fit the tunnel shapes and the main cavern. Sixteen primitives were used to fit the tunnels' shapes and 59 primitives were used to fit the main cavern shape. Then, the rock mass surrounding the excavations was represented. This was done firstly with the bricks at the same level of the tunnels and then with the bricks below and above the tunnels' level. 
Fig. 16 Structure chart for the FORTRAN program

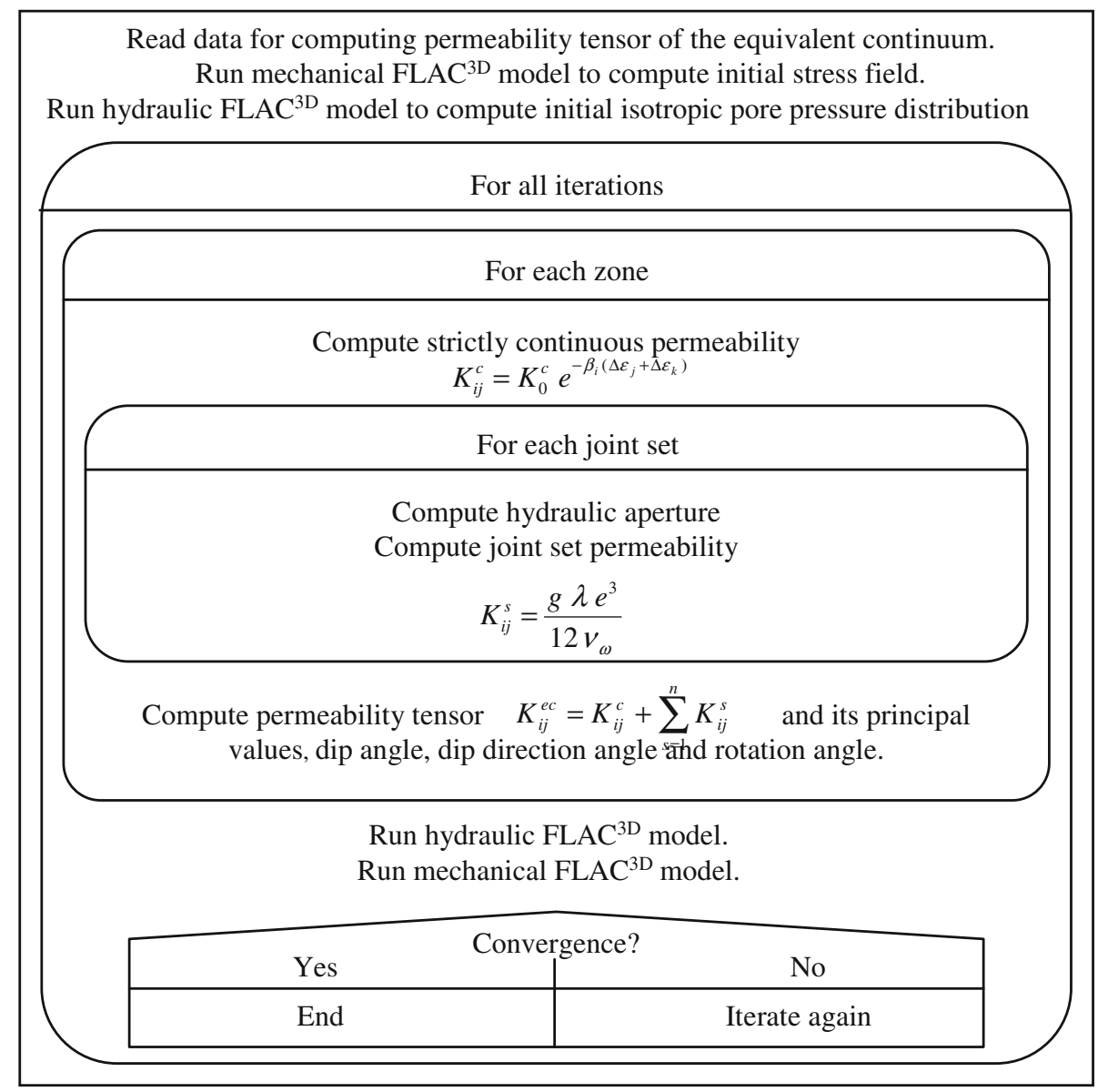

Figure 17 shows the primitives corresponding to the tunnels' level before and after being shaped. The creation of the mesh was thought of as a "fold-and-cut process" in order to obtain a mesh as smooth and as regular as possible (Fig. 18). In total, 641 brick primitives, 93,207 grid points and 81,252 zones were used (Leitão and Lamas 2006b).

\section{Applications of the Numerical Model}

\subsection{Numerical Simulations}

Since the main concern of this study was to capture more realistically the hydraulic behaviour of the rock mass, four different hydraulic analyses of increasing complexity were carried out, namely:

A Continuous medium with isotropic permeability;

B Continuous medium with strain-induced anisotropic permeability;

C Equivalent continuous medium representing one joint set and one-way hydromechanical coupling;

D Equivalent continuous medium representing one joint set and fully hydromechanical coupling.
The reason for this was to investigate the influence on the results obtained, mainly on flow rates, of:

- The large, sub-parallel discontinuities, considered as a joint set;

- Different ways of modelling the rock mass hydraulic behaviour.

In cases $\mathrm{A}$ and $\mathrm{B}$, an initial permeability coefficient $K=10^{-8} \mathrm{~m} / \mathrm{s}$ was adopted in both cases. This corresponds to permeability values common in the technical literature, which are typical for sound granitic rock masses with nearly closed joints. In case $\mathrm{A}$, the hydromechanical coupling parameter $\beta$ was set to zero, i.e. the permeability is constant, whereas in case $\mathrm{B}$, a hydromechanical analysis was performed with $\beta=5,000$, i.e. a strain-dependent permeability.

For cases $\mathrm{C}$ and D, a joint set was considered in order to simulate the main conductive discontinuities found during the infilling (Fig. 12). A dip direction of $75^{\circ}$ and a dip angle of $75^{\circ}$ measured in the global $x y z$ system used in the FLAC $^{3 \mathrm{D}}$ model was adopted. The joint set parameters used in the computation were frequency $\lambda=0.5 \mathrm{~m}^{-1}$, initial aperture $e_{0}=200 \mu \mathrm{m}$, normal stiffness $k_{\mathrm{n}}=100 \mathrm{MPa} / \mathrm{m}$ 
(a)

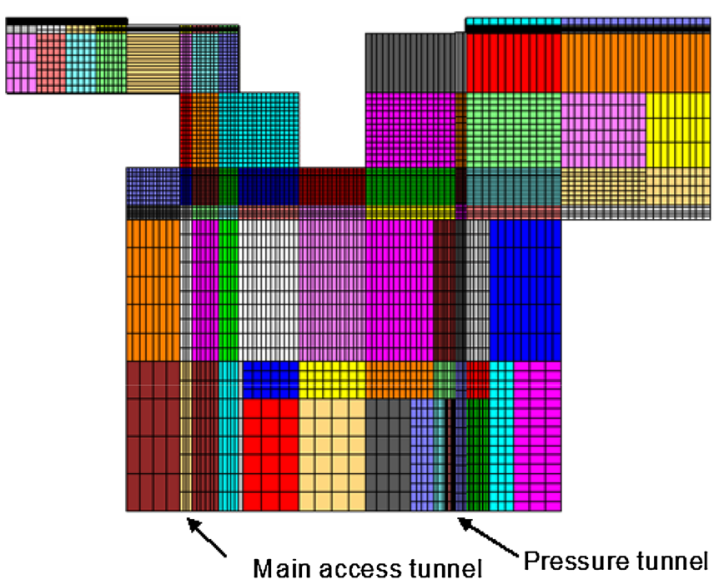

Main access tunnel

(b)

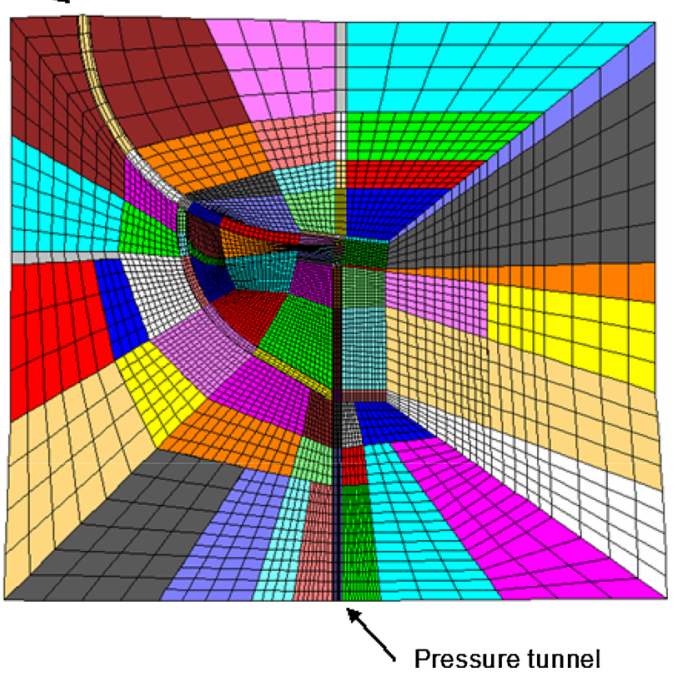

Fig. 17 Tunnels' level: a brick primitives before being shaped, b brick primitives after being shaped

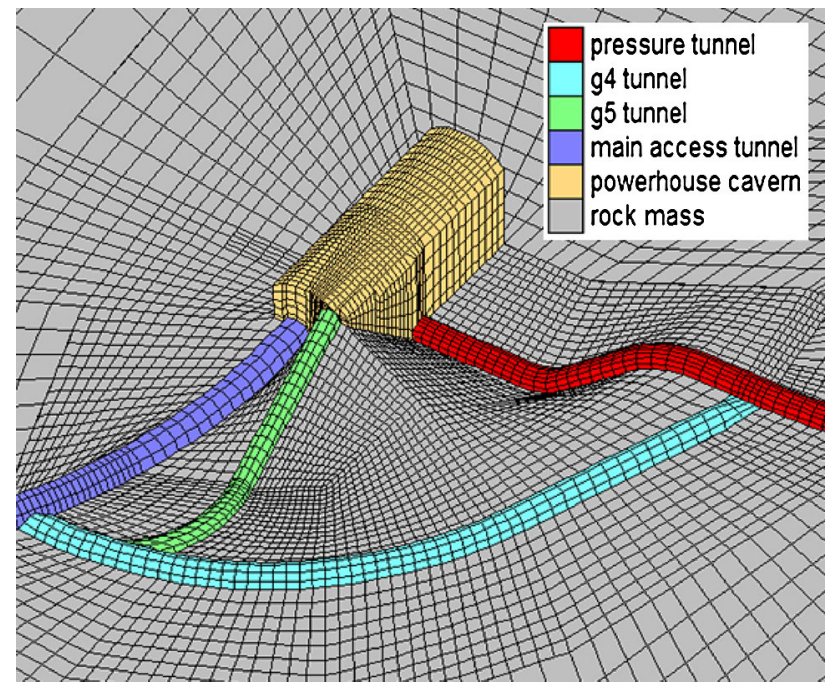

Fig. 18 Grid at the tunnels' level and main powerhouse cavern

and residual aperture $e_{\text {res }}=5 \mu \mathrm{m}$. The choice of values for these parameters presents obvious difficulties. The methodology that was adopted was to fix some of them. The value of the joint normal stiffness was obtained from normal stress tests of granite discontinuities of the same project. The value of joint frequency was based on observation of the conductive discontinuities in adits G4 and G5. For the residual aperture, a very small value, with negligible effect on the results, was adopted. Finally, a parametric analysis was done regarding the value of the joint initial aperture: several values were used, within a range considered to be acceptable, and the value that gave total flow rates in G4 and G5, considered together, closer to the measured values, was chosen.
Additionally, in cases $\mathrm{C}$ and $\mathrm{D}$, a strain-independent $(\beta=0)$ permeability coefficient $K=10^{-9} \mathrm{~m} / \mathrm{s}$ (10 times smaller than in cases A and B) was assumed in order to account for the rock mass behaviour excluding this particular joint set. The difference between cases C and D consists of the way in which the hydromechanical coupling was considered. With reference to the scheme of Fig. 16, case $\mathrm{C}$ was calculated with one iteration only (one-way coupling) and case D with three iterations, when convergence of the hydromechanical iterative process (see Fig. 16) was reached.

To determine the boundary conditions, it was considered that seepage was established from the pressure tunnel into the rock mass during the pressurisation of the tunnel, thus, establishing unconfined flow conditions. A water pressure of 4.5 MPa was imposed in the pressure tunnel and zero water pressures were imposed in all the other underground openings. Assuming that the external faces were far away from the tunnels, a zero-pressure boundary was adopted for all sides of the domain, except for the side intercepted by the pressure tunnel, where an impermeable boundary was considered.

For the mechanical problem, roller boundary conditions were applied on all sides of the domain except the top one, where the weight of the overburden was applied. The rock mass was considered as an elastic material, with Young's modulus $E=30 \mathrm{GPa}$, Poisson's ratio $v=0.15$ and unit weight $\gamma=0.027 \mathrm{MN} / \mathrm{m}^{3}$, which are considered to be representative for the geotechnical zone ZG1 (see Table 2). It would also have been possible to use a multi-laminated model for the mechanical simulation, but for the reasons presented in Sect. 4.2.2, the influence of the discontinuities was not considered to be relevant for this analysis. 


\subsection{Analysis of the Modelling Results}

In order to compute the initial mechanical conditions, the analysis was divided into two stages. In the first stage, the model was brought to a pre-excavation stress state that corresponds to the results of the in situ stress measurement tests: a vertical stress $\sigma_{z z}$ equal to the self-weight, a horizontal stress parallel to the pressure tunnel (normal to the powerhouse longitudinal axis) $\sigma_{x x}=2 \sigma_{z z}$ and a horizontal stress normal to the pressure tunnel $\sigma_{y y}=\sigma_{z z}$. These initial in situ stresses were assigned as initial applied values to the entire model and the fixed lateral boundaries automatically ensure the horizontal equilibrium. In the vertical direction, both equilibrium and the gravitational gradient were satisfied by setting the density of the rock, the gravitational acceleration and the weight of the overlying rock compatible with the applied vertical stress. In the second stage, the tunnels and the cavern were excavated. Figure 19 shows the contour plots of $\sigma_{z z}$ on a vertical plane intersecting the three adits.

Figures 20a, 21a and 22a represent the contours of the vertical component of the permeability tensor, $K_{z z}$, for cases $\mathrm{B}, \mathrm{C}$ and $\mathrm{D}$. The contour colours are graded from blue (minimum value) to red (maximum value). Only the vertical permeability plots are shown, because the main intention is to illustrate how the permeability varies in the several cases that were analysed. Figures $20 \mathrm{~b}, 21 \mathrm{~b}$ and $22 \mathrm{~b}$ are plots of the computed pore pressure distributions around the pressure tunnel for cases B, C and D.

As a consequence of the excavation of the pressure tunnel, the hoop stresses and strains increase from the original pre-excavation values, and the radial stresses and strains decrease. The consequence of this when a straindependent permeability is considered (case B) can be visualised in Fig. 20a. Immediately above and below the tunnel boundary, the vertical permeability $K_{z z}$ (in the radial direction) decreases from its initial value of $10^{-8} \mathrm{~m} / \mathrm{s}$ to a minimum of $0.49 \times 10^{-8} \mathrm{~m} / \mathrm{s}$. Conversely, in the vicinity of tunnels walls, the vertical permeability $K_{z z}$ (in the hoop direction) increases to $2.40 \times 10^{-8} \mathrm{~m} / \mathrm{s}$.

Since the radial permeability is approximately constant around the tunnel, the pore pressure distribution resulting from an internal pressure in the tunnel presents radial symmetry, as can be seen in Fig. 20b. However, the decrease in the radial permeability values when a straindependent permeability is considered results in more concentrated pore pressure contours in the vicinity of the pressure tunnel boundary.

In the permeability and the pore pressure contours for case C (Fig. 21), the major influence of the conductive joint set is clearly illustrated. The orientation of the joint set is visible from the shapes of the contours, and the maximum value of the permeability is over 30 times higher than in case B.
In case D (Fig. 22), after convergence of the hydromechanical iterative process is reached, the final results maintained the same trend as in case $\mathrm{C}$, with the dominant influence of the conductive joint set. The maximum permeability increased by nearly $50 \%$ and the spacing of the pore pressure contours is higher. This shows that the consideration of the hydromechanical coupling has an important influence on the results.

The calculated flow rates into adit G4, adit G5 and the main access tunnel are presented in Table 3 for the four cases. In order to enable the comparison with the overall flow rates into G4 and G5 represented in Fig. 11, they are also shown in the table. The calculated flow rates into the powerhouse were negligible.

In all calculation cases, most of the water that flows from the pressure tunnel is infiltrated into adit G4. This is the closest adit to the pressure tunnel and, as could be expected, it functions as a large drain in the rock mass that collects nearly all the water. The flow rate into adit G4 is $40 \%$ smaller in case B than in case A, because of the decrease in the radial permeability that results from the consideration of a strain-dependent permeability.

In cases $\mathrm{C}$ and $\mathrm{D}$, totally different values of the flow rates were computed, owing to the dominant contribution of the conductive joint set for the overall hydraulic behaviour of the rock mass. The flow rates into adit G5 and into the main access tunnel are no longer negligible, but they are nearly one order of magnitude smaller than that into adit G4. In case C, the calculated flow rates infiltrated into adit G4 are approximately 40 times higher than in cases A and B. When full hydromechanical coupling is considered in case $\mathrm{D}$, the increase in the permeability around the adits during the coupling process also results in an increase in the flow rates, which, in this case, is of about 10-15\% when compared with case C.

\subsection{Comparison with the Observed Behaviour}

In order to compare the calculated with the measured flow rates, it must be borne in mind that, due to the grouting works that were carried out around adit G4 during the infilling in order to control the infiltrations, the effect of main drain, which, in the beginning of the infilling was being performed by G4, was partially transferred to G5, as was explained in detail in Sect. 3.2. Having this in mind, comparisons between the values of the measured infiltrations and the flow rates calculated with the numerical model can only be done for the total water infiltrations into adits G4 + G5, since this transfer of flow from G4 to G5 due to grouting was not considered in the model.

The total infiltrations measured in adits G4 and G5 at the end of the first infilling was approximately equal, with a value of around $4 \mathrm{l} / \mathrm{s}$, which makes a total of $8 \mathrm{l} / \mathrm{s}$ in the 

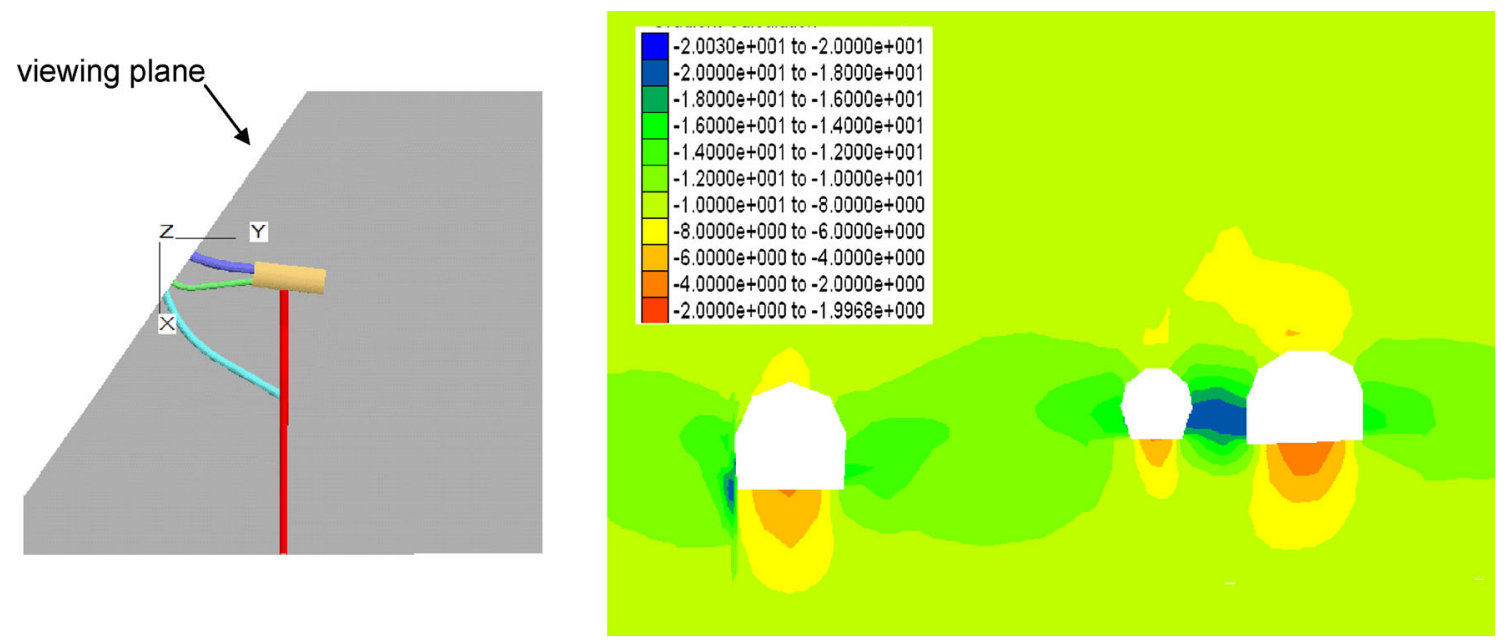

Fig. 19 Contour plots of $\sigma_{z z}$ on a vertical plane intersecting the three adits

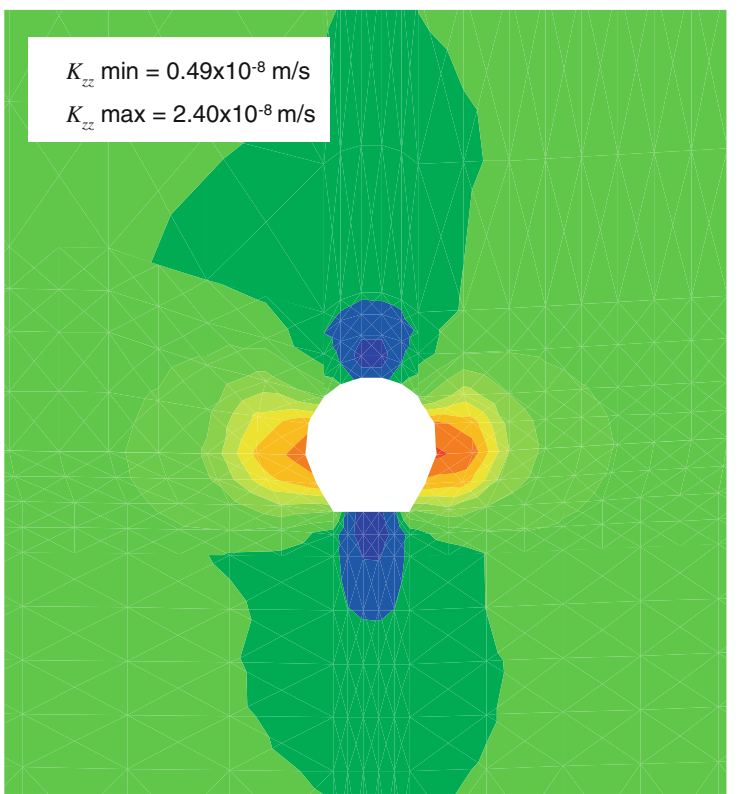

(a)

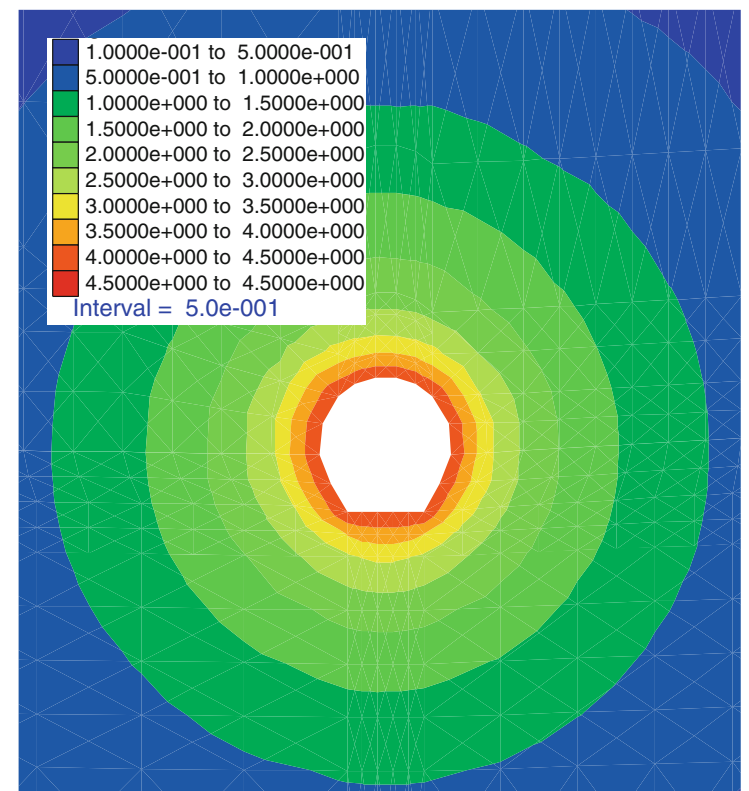

(b)

Fig. 20 Case B: a vertical permeability $K_{z z}$ contours, b pore pressure contours (in MPa)

two adits. The slight flow rate decrease after completion of the first infilling was due to the grouting works and natural obstruction of the joints. In the hydromechanical calculations presented in the previous section, with consideration of the effect of the joint set, the total calculated flow rate into these tunnels was 9.44 and $11.64 \mathrm{l} / \mathrm{s}$, which are values that can be considered as being relatively close to the measured flow rate.

When the rock mass was considered as an equivalent continuum, in cases A and B, with permeability values typical for sound granitic rock masses, the calculated flow rates were 30-50 times lower than the measured ones. In other words, in order to calculate flow rates similar to the measured ones, an isotropic permeability coefficient $K=5 \times 10^{-7} \mathrm{~m} / \mathrm{s}$ would have to be considered for the rock mass in order to give the same flow rates, which is clearly a large and unrealistic value.

From the results obtained, it can be concluded that consideration of the main conductive joint set was essential for modelling the rock mass hydraulic behaviour. Without its consideration, the measured flow rates could not be explained. Consideration of this joint set in the model allowed explaining the measured flow rates. With the properties that were assumed, the calculated flow rates into adits G4 and G5, considered together, match reasonably well the measured values. 


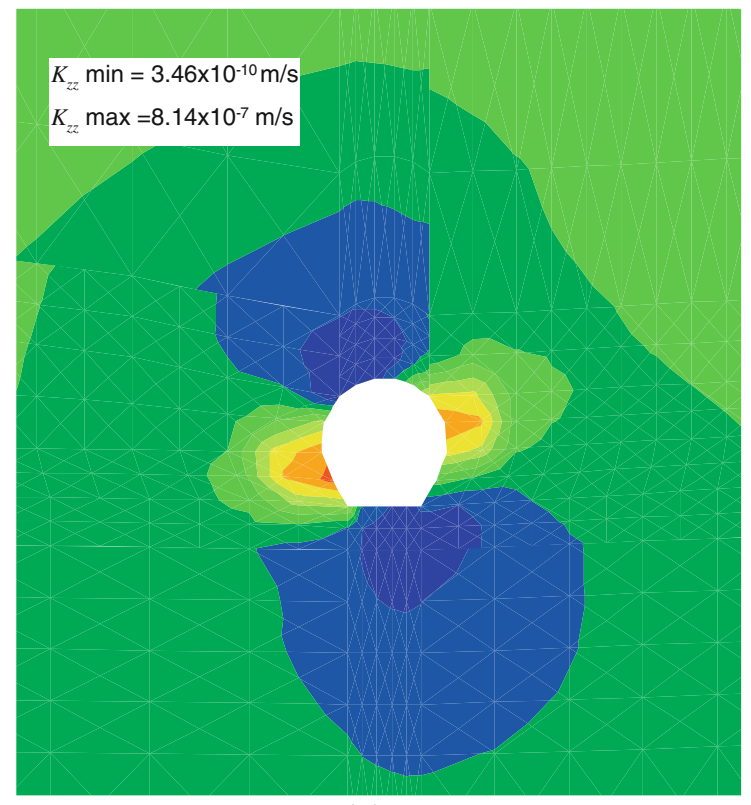

(a)

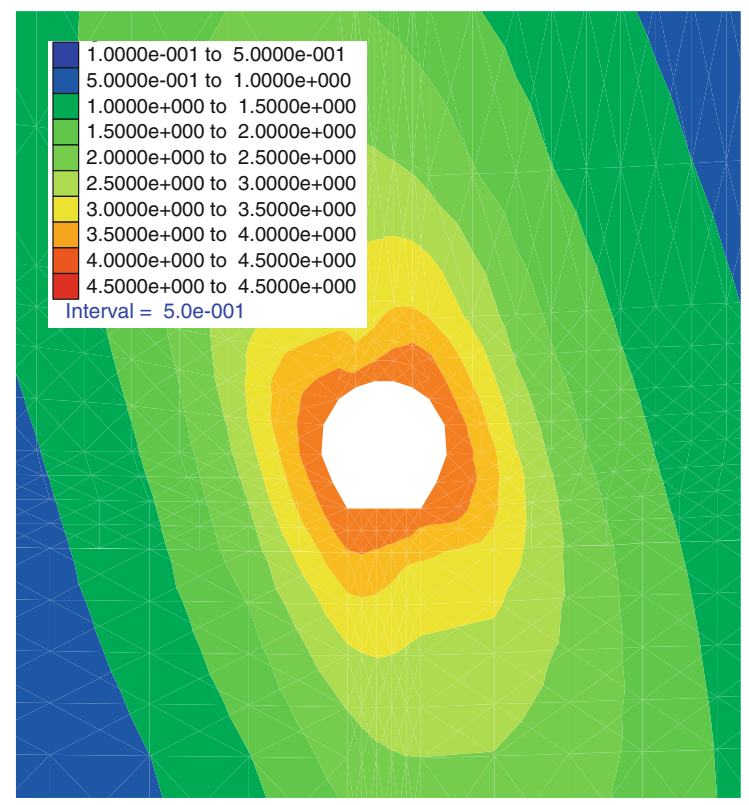

(b)

Fig. 21 Case C: a vertical permeability $K_{z z}$ contours, b pore pressure contours (in MPa)

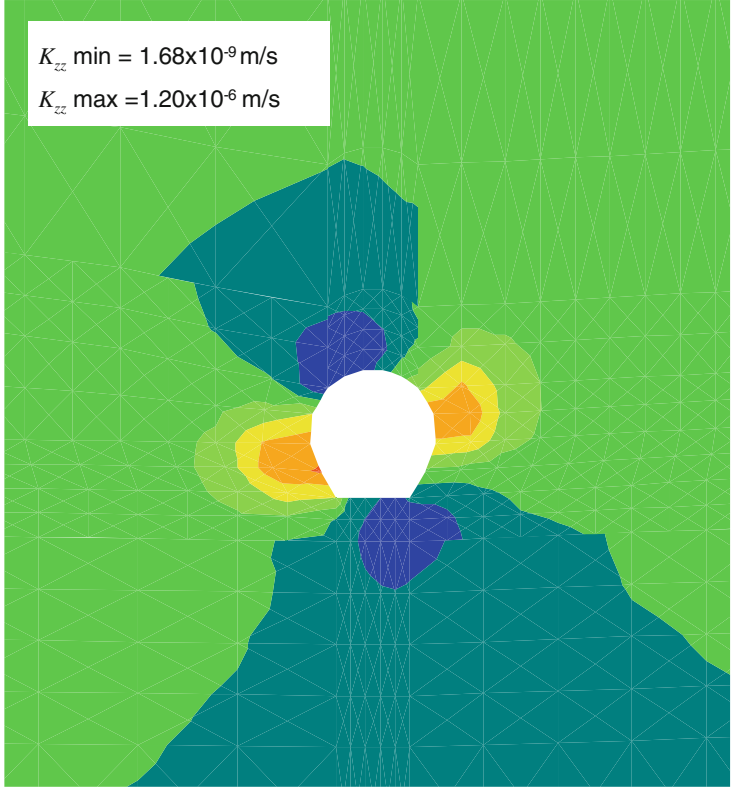

(a)

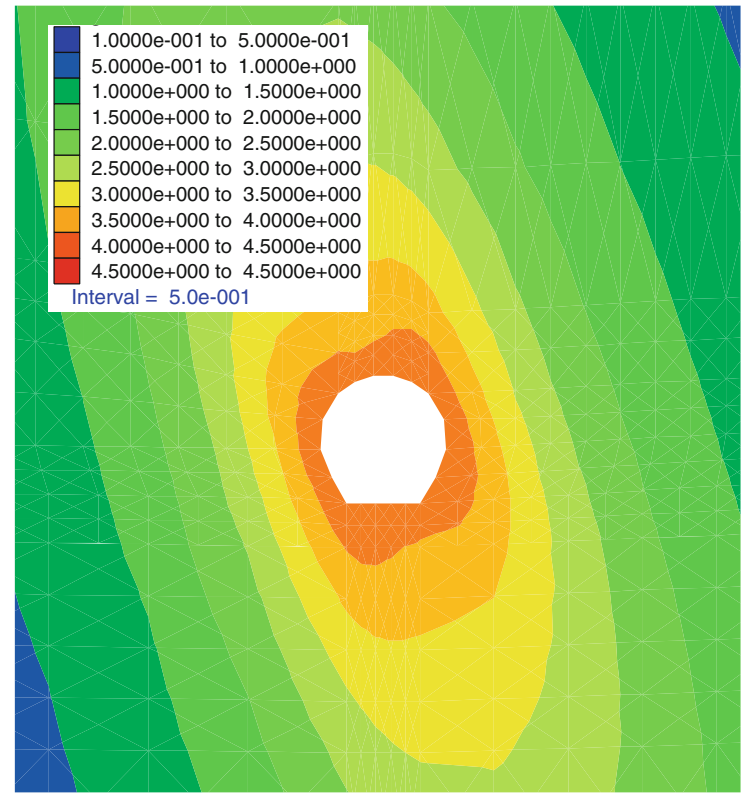

(b)

Fig. 22 Case D: a vertical permeability $K_{z z}$ contours, b pore pressure contours (in MPa)

\section{Conclusions and Discussion}

An "unlined" solution was adopted for the Venda Nova II pressure tunnels, owing to the advantages that were identified, and taking into consideration the good rock mass quality, from both the mechanical and the hydraulic points of view. The rock mass behaviour during the first infilling showed that the geological discontinuities were the key feature that controlled the percolation from the headrace pressure tunnel into the rock mass and the infiltrations in several neighbouring openings. It is worthwhile to note that other geological discontinuities, with important impervious filling, also performed an important role as impermeable barriers.

The existence of a "First Infilling Procedure", including the monitoring of the underground structures and the 
Table 3 Flow rates into adits G4 and G5 and into the main access tunnel

\begin{tabular}{lllll}
\hline Case & \multicolumn{2}{l}{ Flow rate $(1 / \mathrm{s})$} & & \\
\cline { 2 - 5 } & $\mathrm{G} 4$ & $\mathrm{G} 5$ & $\mathrm{G} 4+$ G5 & Main access tunnel \\
\hline A-isotropic, constant permeability & 0.26 & - & 0.26 & - \\
B-strain-dependent permeability & 0.16 & - & 0.16 & - \\
C-multi-laminated, coupled one-way & 8.35 & 1.09 & 9.44 & 1.26 \\
D-multi-laminated, fully coupled & 10.5 & 1.14 & 11.64 & 1.25 \\
\hline
\end{tabular}

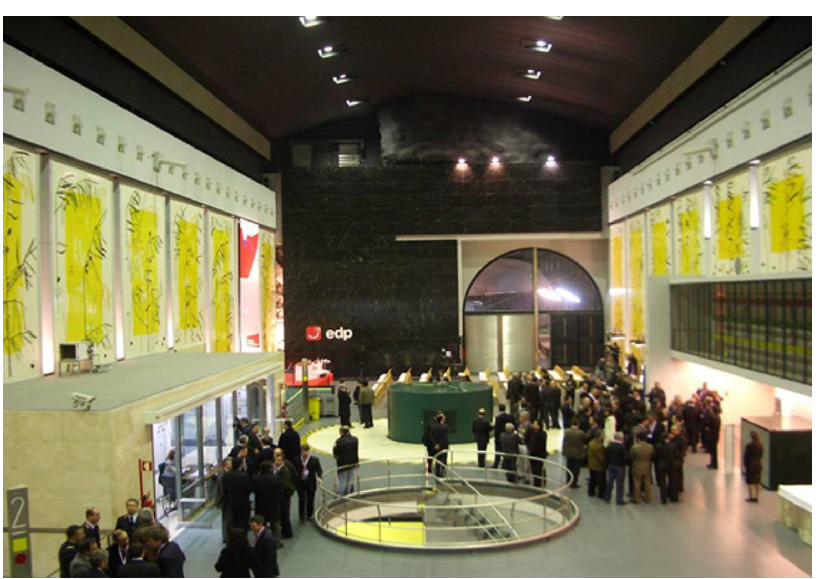

Fig. 23 The Venda Nova II powerhouse has been operating since 2005

surface, was fundamental for the success of the infilling operation in order to guide the decisions that had to be taken in the several infilling stages. During this process, it was necessary to decide when and where to make the several kinds of treatments in order to reduce leakage. When the amount of leakage was considered to be acceptable, the decision was made to stop treatments and to live with drainage of this water. During the infilling phase, the observation of the surface conditions and monitoring of the water table showed no impacts on it. The first infilling took place in December 2004 and the Venda Nova II powerhouse (Fig. 23) has been operating since 2005. As expected, the leakage reduced with time.

The first infilling of the Venda Nova II hydraulic conduit is a well-documented case study in a well-characterised rock mass, where a systematic monitoring system was implemented, which supplied a wealth of results that are not commonly available in the technical literature for similar projects. The measured flow rates revealed relatively high leakage into some adits near the pressure tunnel. These facts constituted the motivation for the deeper study of the hydraulic behaviour of the rock mass during the first infilling, which was performed after the scheme was in normal operation.

A formulation was implemented in the FLAC $^{3 \mathrm{D}}$ numerical model that relates permeability with strain for the continuum, and allows considering the influence of joint sets in the hydraulic behaviour by means of the multilaminated medium concept. Special effort was undertaken to reveal the influence of the presence of several, nearly parallel, large conducting discontinuities. An iterative procedure was developed in order to consider the hydromechanical coupling.

Different ways to simulate the rock mass hydromechanical behaviour were considered. The results were analysed and compared with the flow rates measured during the infilling. The prediction of flow rates by numerical modelling in media where flow takes place through discontinuities is a rather difficult exercise. However, the main conclusion of the numerical analysis is that the large, sub-parallel, conductive joints, which played a dominant role in the hydraulic behaviour of the rock mass, could be adequately simulated as a joint set, using a multi-laminate approach and a hydromechanical model. The calculated results allowed to explain the measured flow rates.

Acknowledgments The permission of Energies of Portugal (EDP) to use the data related to the Venda Nova II project is acknowledged.

\section{References}

Alves L, Resende M, Plasencia N, Ribeiro V (1997) Optimizing the layout of the Venda Nova II pumped storage scheme. In: Hydropower and Dams (ed) Proceedings of "Hydropower into the next century", Portorož, Slovenia, September 1997. Aqua Media International, Sutton, Surrey, UK

Cotelo Neiva JM, Plasencia NS, Lima C (2000) Geological and geotechnical characteristics of the rock mass of the Venda Nova II hydraulic circuit. In: Proceedings of the 7th Portuguese geotechnical congress, Porto, Portugal, pp 113-122 (in Portuguese)

Hidrorumo (1997) Venda Nova II-repowering of the Venda Nova hydroelectric scheme: design documents, vol II. Hidrorumo, Projecto e Gestão S.A., Porto (in Portuguese)

International Society for Rock Mechanics (ISRM) (1978) Suggested methods for the quantitative description of discontinuities in rock masses. Int J Rock Mech Min Sci Geomech Abstr 15:319-368

International Society for Rock Mechanics (ISRM) (1981) Basic geotechnical description of rock masses. Int J Rock Mech Min Sci Geomech Abstr 18:85-110

Itasca Consulting Group (2002) FLAC $^{3 \mathrm{D}}$ —fast Lagrangian analysis of continua in 3 dimensions, ver. 2.1. User's manual. Itasca Consulting Group, Inc., Minneapolis, MN, USA

Laboratório Nacional de Engenharia Civil (LNEC) (2003a) Venda Nova II scheme. Determination of the stress state. LNEC report 371/03-NFOS. LNEC, Lisbon, Portugal (in Portuguese) 
Laboratório Nacional de Engenharia Civil (LNEC) (2003b) Plan for the first infilling and beginning of operation of the Venda Nova II powerhouse and hydraulic circuit. LNEC report 131/04NFOS. LNEC, Lisbon, Portugal (in Portuguese)

Lamas LN (1993) Contributions to understanding the hydromechanical behaviour of pressure tunnels. $\mathrm{PhD}$ thesis, Imperial College, London, UK

Lamas LN, Sousa LR (1993) The use of a hydromechanical numerical model to understand the behaviour of pressure tunnels and shafts. In: Ribeiro L, Sousa E, Grossmann NF (eds) Proceedings of Eurock '93: safety and environmental issues in rock engineering, Lisbon, Portugal, June 1993. Balkema, Rotterdam, the Netherlands, pp 961-968

Leitão NS, Lamas LN (2006a) Implementation of a 3D multilaminated hydromechanical model for analysis of an unlined high pressure tunnel. In: Yao ZH, Yuan MW, Chen YQ (eds) Proceedings of EPMESC X: computational methods in engineering and science, Sanya, Hainan, China, August 2006. Tsinghua University Press \& Springer, pp 274-283

Leitão NS, Lamas LN (2006b) Modeling of the high pressure circuit of the Venda Nova II hydroelectric scheme. In: Hart, Varona (eds) Proceedings of 4th international FLAC symposium on numerical modeling in geomechanics, Madrid, Spain, May 2006. Itasca Consulting Group, Inc., Minneapolis, MN, USA

Leitão NS, Lamas LN, Esteves C (2005) Numerical modelling of the behaviour of the Venda Nova II hydraulic circuit-description of the model. In: Gomes C, Roque L, Pardo B (eds) Proceedings of the Portuguese-Spanish geotechnical congress, SPG, Lisbon, Portugal, pp. 295-302 (in Portuguese)

Lima C, Resende M, Plasencia N, Esteves C (2002) Venda Nova II hydroelectric scheme powerhouse geotechnics and design. ISRM News J 7(2):37-41

Oliveira MA, Ribeiro V, Apolinário V, Costa JA (2004) The Venda Nova II pumped storage scheme. Int J Hydropower Dams 11(5):88-92

Plasencia NS (2003) Contributions of engineering geology for conception and design of underground structures. MSc thesis, Technical University of Lisbon, Lisbon, Portugal (in Portuguese)

Plasencia NS (2008) Hydraulic tunnels of EDP Produção-Venda Nova II repowering scheme. In: Proceedings of the 2 nd seminar on Portuguese commission of tunnels, LNEC, Lisbon, Portugal (in Portuguese)

Plasencia NS, Coelho MJ, Lima C, Fialho L (2000) Contribution of seismic tomography to the characterization of Venda Nova II powerhouse rock mass. In: Proceedings of the 7th Portuguese geotechnical congress, Porto, Portugal

Rutqvist J, Stephansson O (2003) The role of hydromechanical coupling in fractured rock engineering. Hydrogeol J 11:7-40

Wei L (1992) Numerical studies of the hydro-mechanical behaviour of jointed rocks. PhD thesis, Imperial College, London, UK

Zienkiewicz OC, Pande GN (1977) Time-dependent multilaminate model of rocks - a numerical study of deformation and failure of rock masses. Int J Numer Anal Methods Geomech 1:219-247 